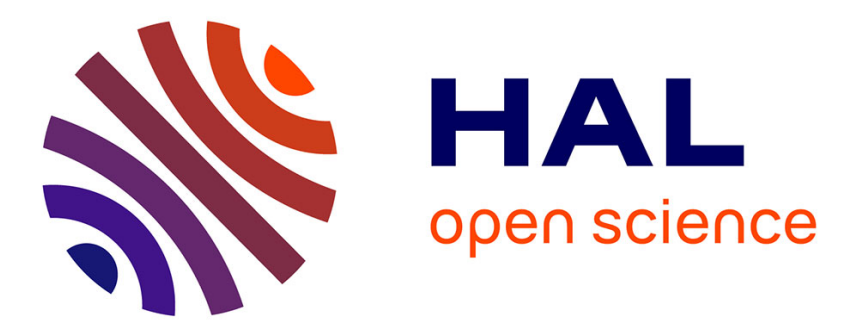

\title{
Densité pulmonaire et quantification vasculaire tomodensitométrique dans l'hypertension pulmonaire associée aux pneumopathies interstitielles diffuses fibrosantes
}

Kim Valette

\section{To cite this version:}

Kim Valette. Densité pulmonaire et quantification vasculaire tomodensitométrique dans l'hypertension pulmonaire associée aux pneumopathies interstitielles diffuses fibrosantes. Sciences du Vivant [q-bio]. 2020. dumas-03102325

\section{HAL Id: dumas-03102325 https://dumas.ccsd.cnrs.fr/dumas-03102325}

Submitted on 7 Jan 2021

HAL is a multi-disciplinary open access archive for the deposit and dissemination of scientific research documents, whether they are published or not. The documents may come from teaching and research institutions in France or abroad, or from public or private research centers.
L'archive ouverte pluridisciplinaire HAL, est destinée au dépôt et à la diffusion de documents scientifiques de niveau recherche, publiés ou non, émanant des établissements d'enseignement et de recherche français ou étrangers, des laboratoires publics ou privés. 
Densité pulmonaire et quantification vasculaire tomodensitométrique dans l'hypertension pulmonaire associée aux pneumopathies interstitielles diffuses fibrosantes.

\title{
T H E S E A A R T I C L E
}

Présentée et publiquement soutenue devant

\section{LA FACULTÉ DES SCIENCES MEDICALES ET PARAMEDICALES}

\author{
DE MARSEILLE
}

Le 10 Décembre 2020

Par Madame Kim VALETTE

Née le 13 décembre 1991 à Toulon $(83)$

Pour obtenir le grade de Docteur en Médecine

D.E.S. de PNEUMOLOGIE

Membres du Jury de la Thèse :

Madame le Professeur REYNAUD-GAUBERT Martine

Président

Monsieur le Professeur CHANEZ Pascal

Assesseur

Monsieur le Professeur GAUBERT Jean-Yves

Assesseur

Monsieur le Docteur COIFFARD Benjamin

Directeur 

Densité pulmonaire et quantification vasculaire tomodensitométrique dans l'hypertension pulmonaire associée aux pneumopathies interstitielles diffuses fibrosantes.

\title{
T H E S E A A R T I C L E
}

Présentée et publiquement soutenue devant

\section{LA FACULTÉ DES SCIENCES MEDICALES ET PARAMEDICALES}

\author{
DE MARSEILLE
}

Le 10 Décembre 2020

Par Madame Kim VALETTE

Née le 13 décembre 1991 à Toulon $(83)$

Pour obtenir le grade de Docteur en Médecine

D.E.S. de PNEUMOLOGIE

Membres du Jury de la Thèse :

Madame le Professeur REYNAUD-GAUBERT Martine

Président

Monsieur le Professeur CHANEZ Pascal

Assesseur

Monsieur le Professeur GAUBERT Jean-Yves

Assesseur

Monsieur le Docteur COIFFARD Benjamin

Directeur 


\section{FACULTÉ DES SCIENCES MÉDICALES \& PARAMÉDICALES}

\section{Doyen}

Vice-Doyen aux affaires générales

Vice-Doyen aux professions paramédicales

Conseiller

\section{Assesseurs :}

\section{aux études}

$>$ à la recherche

$>$ à l'unité mixte de formation continue en santé

$>$ pour le secteur NORD

$>$ Groupements Hospitaliers de territoire

$>$ aux masters
Pr. Georges LEONETTI

Pr. Patrick DESSI

Pr. Philippe BERBIS

Pr. Patrick VILLANI

\section{Chargés de mission :}

$>$ sciences humaines et sociales

$>$ relations internationales

$>$ DU/DIU

$>$ DPC, disciplines médicales \& biologiques

$>$ DPC, disciplines chirurgicales

\section{ÉCOLE DE MEDECINE}

\section{Directeur}

\section{Chargés de mission}

- PACES - Post-PACES

- DFGSM

- DFASM

- DFASM

- Préparation aux ECN

- DES spécialités

- DES stages hospitaliers

- DES MG

- Démographie médicale

- Etudiant
Pr. Pierre LE COZ
Pr. Stéphane RANQUE
Pr. Véronique VITTON
Pr. Frédéric CASTINETTI
Dr. Thomas GRAILLON

$\begin{array}{ll}: & \text { Pr. Pierre LE COZ } \\ : & \text { Pr. Stéphane RANQUE } \\ : & \text { Pr. Véronique VITTON } \\ : & \text { Pr. Frédéric CASTINETTI } \\ : & \text { Dr. Thomas GRAILLON }\end{array}$




\section{ÉCOLE DE DE MAIEUTIQUE}

\section{Directrice}

\section{Chargés de mission}

- $1^{\text {er }}$ cycle

- $2^{\text {ème }}$ cycle
Madame Carole ZAKARIAN

Madame Estelle BOISSIER

Madame Cécile NINA

\section{ÉCOLE DES SCIENCES DE LA RÉADAPTATION}

Directeur

\section{Chargés de mission}

- Masso- kinésithérapie $1^{\mathrm{er}}$ cycle

- Masso-kinésithérapie $2^{\text {ème }}$ cycle

- Mutualisation des enseignements
Monsieur Philippe SAUVAGEON

Madame Béatrice CAORS

Madame Joannie HENRY

Madame Géraldine DEPRES

\section{ÉCOLE DES SCIENCES INFIRMIERES}

Directeur

\section{Chargés de mission}

- Chargée de mission

- Chargé de mission
Monsieur Sébastien COLSON

Madame Sandrine MAYEN RODRIGUES Monsieur Christophe ROMAN 


\section{PROFESSEURS HONORAIRES}

MM AGOSTINI Serge

ALDIGHIERI René

ALESSANDRINI Pierre

ALLIEZ Bernard

AQUARON Robert

ARGEME Maxime

ASSADOURIAN Robert

AUFFRAY Jean-Pierre

AUTILLO-TOUATI Amapola

AZORIN Jean-Michel

BAILLE Yves

BARDOT Jacques

BARDOT André

BERARD Pierre

BERGOIN Maurice

BERLAND Yvon

BERNARD Dominique

BERNARD Jean-Louis

BERNARD Jean-Paul

BERNARD Pierre-Marie

BERTRAND Edmond

BISSET Jean-Pierre

BLANC Bernard

BLANC Jean-Louis

BOLLINI Gérard

BONGRAND Pierre

BONNEAU Henri

BONNOIT Jean

BORY Michel

BOTTA Alain

BOTTA-FRIDLUND Danielle

BOURGEADE Augustin

BOUVENOT Gilles

BOUYALA Jean-Marie

BREMOND Georges

BRICOT René

BRUNET Christian

BUREAU Henri

CAMBOULIVES Jean

CANNONI Maurice

CARTOUZOU GuY

CAU Pierre

CHABOT Jean-Michel

CHAMLIAN Albert

CHARPIN Denis

CHARREL Michel
MM DEVRED Philippe

DJIANE Pierre

DONNET Vincent

DUCASSOU Jacques

DUFOUR Michel

DUMON Henri

ENJALBERT Alain

FAUGERE Gérard

FAVRE Roger

FIECHI Marius

FARNARIER Georges

FIGARELLA Jacques

FONTES Michel

FRANCES Yves

FRANCOIS Georges

FUENTES Pierre

GABRIEL Bernard

GALINIER Louis

GALLAIS Hervé

GAMERRE Marc

GARCIN Michel

GARNIER Jean-Marc

GAUTHIER André

GERARD Raymond

GEROLAMI-SANTANDREA André

GIUDICELLI Sébastien

GOUDARD Alain

GOUIN François

GRILLO Jean-Marie

GRIMAUD Jean-Charles

GRISOLI François

GROULIER Pierre

HADIDA/SAYAG Jacqueline

HASSOUN Jacques

HEIM Marc

HOUEL Jean

HUGUET Jean-François

JAQUET Philippe

JAMMES Yves

JOUVE Paulette

JUHAN Claude

JUIN Pierre

KAPHAN Gérard

KASBARIAN Michel

KLEISBAUER Jean-Pierre

LACHARD Jean 
CHAUVEL Patrick

CHOUX Maurice

CIANFARANI François

CLAVERIE Jean-Michel

CLEMENT Robert

COMBALBERT André

CONTE-DEVOLX Bernard

CORRIOL Jacques

COULANGE Christian

CURVALE Georges

DALMAS Henri

DE MICO Philippe

DELPERO Jean-Robert

DESSEIN Alain

DELARQUE Alain

DEVIN Robert

MM MICHOTEY Georges

MIRANDA François

MONFORT Gérard

MONGES André

MONGIN Maurice

MUNDLER Olivier

NAZARIAN Serge

NICOLI René

NOIRCLERC Michel

OLMER Michel

OREHEK Jean

PAPY Jean-Jacques

PAULIN Raymond

PELOUX Yves

PENAUD Antony

PENE Pierre

PIANA Lucien

PICAUD Robert

PIGNOL Fernand

POGGI Louis

POITOUT Dominique

PONCET Michel

POUGET Jean

PRIVAT Yvan

QUILICHINI Francis

RANQUE Jacques

RANQUE Philippe

RICHAUD Christian

RIDINGS Bernard

ROCHAT Hervé

ROHNER Jean-Jacques

ROUX Hubert
LAFFARGUE Pierre

LAUGIER René

LE TREUT Yves

LEVY Samuel

LOUCHET Edmond

LOUIS René

LUCIANI Jean-Marie

MAGALON Guy

MAGNAN Jacques

MALLAN- MANCINI Josette

MALMEJAC Claude

MARANINCHI Dominique

MARTIN Claude

MATTEI Jean François

MERCIER Claude

METGE Paul

VANUXEM Paul

VERVLOET Daniel

VIALETTES Bernard

WEILLER Pierre-Jean 
ROUX Michel

RUFO Marcel

SAHEL José

SALAMON Georges

SALDUCCI Jacques

SAMBUC Roland

SAN MARCO Jean-Louis

SANKALE Marc

SARACCO Jacques

SARLES Jacques

SARLES - PHILIP Nicole

SASTRE Bernard

SCHIANO Alain

SCOTTO Jean-Claude

SEBAHOUN Gérard

SEITZ Jean-François

SERMENT Gérard

SOULAYROL René

STAHL André

TAMALET Jacques

TARANGER-CHARPIN Colette

THIRION Xavier

THOMASSIN Jean-Marc

UNAL Daniel

VAGUE Philippe

VAGUE/JUHAN Irène 


\section{EMERITAT}

2008

M. le Professeur

LEVY Samuel

$31 / 08 / 2011$

Mme le Professeur

JUHAN-VAGUE Irène

$31 / 08 / 2011$

M. le Professeur

PONCET Michel

$31 / 08 / 2011$

M. le Professeur

KASBARIAN Michel

$31 / 08 / 2011$

M. le Professeur

ROBERTOUX Pierre

$31 / 08 / 2011$

2009

M. le Professeur

DJIANE Pierre

$31 / 08 / 2011$

M. le Professeur

VERVLOET Daniel

$31 / 08 / 2012$

2010

M. le Professeur

MAGNAN Jacques

$31 / 12 / 2014$

2011

M. le Professeur

DI MARINO Vincent

$31 / 08 / 2015$

M. le Professeur

MARTIN Pierre

$31 / 08 / 2015$

M. le Professeur

METRAS Dominique

$31 / 08 / 2015$

\section{2}

M. le Professeur

AUBANIAC Jean-Manuel

$31 / 08 / 2015$

M. le Professeur

BOUVENOT Gilles

$31 / 08 / 2015$

M. le Professeur

M. le Professeur

M. le Professeur

M. le Professeur

M. le Professeur

2013

M. le Professeur

M. le Professeur

M. le Professeur

M. le Professeur

M. le Professeur

M. le Professeur

M. le Professeur

M. le Professeur

CAMBOULIVES Jean

$31 / 08 / 2015$

FAVRE Roger

$31 / 08 / 2015$

MATTEI Jean-François

$31 / 08 / 2015$

OLIVER Charles

$31 / 08 / 2015$

VERVLOET Daniel

$31 / 08 / 2015$

BRANCHEREAU Alain

$31 / 08 / 2016$

CARAYON Pierre

$31 / 08 / 2016$

COZZONE Patrick

$31 / 08 / 2016$

DELMONT Jean

$31 / 08 / 2016$

HENRY Jean-François

$31 / 08 / 2016$

LE GUICHAOUA Marie-Roberte

$31 / 08 / 2016$

RUFO Marcel

$31 / 08 / 2016$

SEBAHOUN Gérard

$31 / 08 / 2016$

\section{4}

M. le Professeur

FUENTES Pierre

$31 / 08 / 2017$

M. le Professeur

GAMERRE Marc

$31 / 08 / 2017$

MAGALON Guy

$31 / 08 / 2017$

M. le Professeur

PERAGUT Jean-Claude

$31 / 08 / 2017$

M. le Professeur

WEILLER Pierre-Jean 
FAVRE Roger $31 / 08 / 2018$ OLIVER Charles $31 / 08 / 2018$ SEBBAHOUN Gérard 
M. le Professeur

M. le Professeur

M. le Professeur

M. le Professeur

M. le Professeur

M. le Professeur

2020

M. le Professeur

M. le Professeur

M. le Professeur

M. le Professeur

M. le Professeur

M. le Professeur

M. le Professeur

M. le Professeur

M. le Professeur

M. le Professeur

M. le Professeur

M. le Professeur

M. le Professeur
FAVRE Roger

$31 / 08 / 2020$

FONTES Michel

$31 / 08 / 2020$

MAGALON Guy

$31 / 08 / 2020$

NAZARIAN Serge

$31 / 08 / 2020$

OLIVER Charles

$31 / 08 / 2020$

WEILLER Pierre-Jean

$31 / 08 / 2020$

DELPERO Jean-Robert

$31 / 08 / 2023$

GRIMAUD Jean-Charles

$31 / 08 / 2023$

SAMBUC Roland

$31 / 08 / 2023$

SEITZ Jean-François

$31 / 08 / 2023$

BERLAND Yvon

$31 / 08 / 2022$

CHARPIN Denis

$31 / 08 / 2022$

CLAVERIE Jean-Michel

$31 / 08 / 2022$

FRANCES Yves

$31 / 08 / 2022$

BONGRAND Pierre

$31 / 08 / 2021$

COZZONE Patrick

FAVRE Roger

$31 / 08 / 2021$

$31 / 08 / 2021$

$31 / 08 / 2021$

$31 / 08 / 2021$ 


\section{Honoris causa}

1967

MM. les Professeurs

1974

MM. les Professeurs

1975

MM. les Professeurs

1976

MM. les Professeurs

1977

MM. les Professeurs

M. le Président

1980

MM. les Professeurs

1981

MM. les Professeurs

M. le Professeur

1985

MM. les Professeurs

1986

MM. les Professeurs
DADI (Italie)

CID DOS SANTOS (Portugal)

MAC ILWAIN (Grande-Bretagne)

T.A. LAMBO (Suisse)

O. SWENSON (U.S.A.)

Lord J.WALTON of DETCHANT (Grande-Bretagne)

P. FRANCHIMONT (Belgique)

Z.J. BOWERS (U.S.A.)

C. GAJDUSEK-Prix Nobel (U.S.A.)

C.GIBBS (U.S.A.)

J. DACIE (Grande-Bretagne)

F. HOUPHOUET-BOIGNY (Côte d'Ivoire)

A. MARGULIS (U.S.A.)

R.D. ADAMS (U.S.A.)

H. RAPPAPORT (U.S.A.)

M. SCHOU (Danemark)

M. AMENT (U.S.A.)

Sir A. HUXLEY (Grande-Bretagne)

S. REFSUM (Norvège)

W.H. HENDREN (U.S.A.)

S. MASSRY (U.S.A.)

KLINSMANN (R.D.A.)

E. MIHICH (U.S.A.)

T. MUNSAT (U.S.A.)

LIANA BOLIS (Suisse)

L.P. ROWLAND (U.S.A.) 


\section{8}

MM. les Professeurs

R. BERGUER (U.S.A.)

W.K. ENGEL (U.S.A.)

V. ASKANAS (U.S.A.)

J. WEHSTER KIRKLIN (U.S.A.)

A. DAVIGNON (Canada)

A. BETTARELLO (Brésil)

1989

P. MUSTACCHI (U.S.A.)

1990

MM. les Professeurs

J.G. MC LEOD (Australie)

J. PORTER (U.S.A.)

1991

MM. les Professeurs

J. Edward MC DADE (U.S.A.)

W. BURGDORFER (U.S.A.)

1992

MM. les Professeurs

H.G. SCHWARZACHER (Autriche)

D. CARSON (U.S.A.)

T. YAMAMURO (Japon)

1994

MM. les Professeurs

G. KARPATI (Canada)

W.J. KOLFF (U.S.A.)

1995

MM. les Professeurs

D. WALKER (U.S.A.)

M. MULLER (Suisse)

V. BONOMINI (Italie)

1997

MM. les Professeurs

C. DINARELLO (U.S.A.)

D. STULBERG (U.S.A.)

A. MEIKLE DAVISON (Grande-Bretagne)

P.I. BRANEMARK (Suède)

1998

MM. les Professeurs

O. JARDETSKY (U.S.A.)

1999

MM. les Professeurs

J. BOTELLA LLUSIA (Espagne) 
D. COLLEN (Belgique)

S. DIMAURO (U. S. A.)

2000

MM. les Professeurs

D. SPIEGEL (U. S. A.)

C. R. CONTI (U.S.A.)

2001

MM. les Professeurs

P-B. BENNET (U. S. A.)

G. HUGUES (Grande Bretagne)

J-J. O'CONNOR (Grande Bretagne)

2002

MM. les Professeurs

M. ABEDI (Canada)

K. DAI (Chine)

2003

M. le Professeur

Sir

T. MARRIE (Canada)

G.K. RADDA (Grande Bretagne)

2004

M. le Professeur

M. DAKE (U.S.A.)

2005

M. le Professeur

L. CAVALLI-SFORZA (U.S.A.)

2006

M. le Professeur

A. R. CASTANEDA (U.S.A.)

2007

M. le Professeur

S. KAUFMANN (Allemagne) 
AGOSTINI FERRANDES Aubert

ALBANESE Jacques

ALIMI Yves

AMABILE Philippe

AMBROSI Pierre

ANDRE Nicolas

ARGENSON Jean-Noël

ASTOUL Philippe

ATTARIAN Shahram

AUDOUIN Bertrand

AUQUIER Pascal

AVIERINOS Jean-François

AZULAY Jean-Philippe

BAILLY Daniel

BARLESI Fabrice

BARLIER-SETTI Anne

BARLOGIS Vincent

BARTHET Marc

BARTOLI Christophe

BARTOLI Jean-Michel

BARTOLI Michel

BARTOLOMEI Fabrice

BASTIDE Cyrille

BENSOUSSAN Laurent

BERBIS Philippe

BERBIS Julie

BERDAH Stéphane

BEROUD Christophe

BERTUCCI François

BLAISE Didier

BLIN Olivier

BLONDEL Benjamin

BONIN/GUILLAUME Sylvie

BONELLO Laurent

BONNET Jean-Louis

BOUBLI Léon Surnombre

BOUFI Mourad

BOYER Laurent

BREGEON Fabienne

BRETELLE Florence

BROUQUI Philippe

BRUDER Nicolas

BRUE Thierry

BRUNET Philippe

BURTEY Stéphane

CARCOPINO-TUSOLI Xavier

CASANOVA Dominique

CASTINETTI Frédéric

CECCALDI Mathieu

CHAGNAUD Christophe

CHAMBOST Hervé

CHAMPSAUR Pierre

CHANEZ Pascal

CHARAFFE-JAUFFRET Emmanuelle

CHARREL Rémi

CHAUMOITRE Kathia

CHIARONI Jacques

CHINOT Olivier
CHOSSEGROS Cyrille

COLLART Frédéric

COSTELLO Régis

COURBIERE Blandine

COWEN Didier

CRAVELLO Ludovic

CUISSET Thomas

DA FONSECA David

DAHAN-ALCARAZ Laetitia

DANIEL Laurent

DARMON Patrice

DAVID Thierry

D'ERCOLE Claude

D'JOURNO Xavier

DEHARO Jean-Claude

DELAPORTE Emmanuel

DENIS Danièle

DISDIER Patrick

DODDOLI Christophe

DRANCOURT Michel

DUBUS Jean-Christophe

DUFFAUD Florence

DUFOUR Henry

DURAND Jean-Marc

DUSSOL Bertrand

EBBO Mikaël

EUSEBIO Alexandre

FABRE Alexandre

FAKHRY Nicolas

FELICIAN Olvier

FENOLLAR Florence

FIGARELLA/BRANGER Dominique

FLECHER Xavier

FOUILLOUX Virginie

FOURNIER Pierre-Edouard

FRANCESCHI Frédéric

FUENTES Stéphane

GABERT Jean

GABORIT Bénédicte

GAINNIER Marc

GARCIA Stéphane

GARIBOLDI Vlad

GAUDART Jean

GAUDY-MARQUESTE Caroline

GENTILE Stéphanie

GERBEAUX Patrick

GEROLAMI/SANTANDREA René

GILBERT/ALESSI Marie-Christine

GIORGI Roch

GIOVANNI Antoine

GIRARD Nadine

GIRAUD/CHABROL Brigitte

GONCALVES Anthony

GRANEL/REY Brigitte

GRANVAL Philippe

GREILLIER Laurent

GROB Jean-Jacques

GUEDJ Eric 
PAGANELLI Franck PANUEL Michel Surnombre PAPAZIAN Laurent PAROLA Philippe PARRATTE Sébastien Disponibilité PELISSIER-ALICOT Anne-Laure PELLETIER Jean PERRIN Jeanne PETIT Philippe PHAM Thao PIERCECCHI/MARTI Marie-Dominiqu SCHLEINITZ Nicolas PIQUET Philippe PIRRO Nicolas POINSO François RACCAH Denis RANQUE Stéphane RAOULT Didier Surnombre REGIS Jean REYNAUD/GAUBERT Martine REYNAUD Rachel RICHARD/LALLEMAND Marie-AlethTROPIANO Patrick RICHIERI Raphaëlle

\author{
ROCHE Pierre-Hugues \\ ROCH Antoine \\ ROCHWERGER Richard \\ ROLL Patrice \\ ROSSI Dominique \\ ROSSI Pascal \\ ROUDIER Jean \\ SALAS Sébastien \\ SARLON-BARTOLI Gabrielle \\ SCAVARDA Didier \\ SEBAG Frédéric \\ SIELEZNEFF Igor \\ SIMON Nicolas \\ STEIN Andréas \\ TAIEB David \\ THOMAS Pascal \\ THUNY Franck \\ TREBUCHON-DA FONSECA Agnès \\ TRIGLIA Jean-Michel \\ TSIMARATOS Michel
}

\title{
PROFESSEUR DES UNIVERSITES
}

\author{
ADALIAN Pascal \\ AGHABABIAN Valérie \\ BELIN Pascal \\ CHABANNON Christian \\ CHABRIERE Eric \\ FERON François \\ LE COZ Pierre \\ LEVASSEUR Anthony \\ RANJEVA Jean-Philippe \\ SOBOL Hagay \\ PROFESSEUR CERTIFIE
}

BRANDENBURGER Chantal retraite mars 2021

FRAISSE-MANGIALOMINI Jeanne

PROFESSEUR DES UNIVERSITES ASSOCIE à MI

REVIS Joana

PROFESSEUR DES UNIVERSITES MEDECINE GEN

GENTILE Gaëtan

PROFESSEUR ASSOCIE DE MEDECINE GENERALE ,

\author{
GUIDA Pierre
}


GUIEU Régis

GUIS Sandrine

GUYE Maxime

GUYOT Laurent

GUYS Jean-Michel Surnombre

HABIB Gilbert

HARDWIGSEN Jean

HARLE Jean-Robert

HOUVENAEGHEL Gilles

JACQUIER Alexis

JOURDE-CHICHE Noémie

JOUVE Jean-Luc

KAPLANSKI Gilles

KARSENTY Gilles

KERBAUL François détachement

KRAHN Martin

LAFFORGUE Pierre

LAGIER Jean-Christophe

LAMBAUDIE Eric

LANCON Christophe

LA SCOLA Bernard

LAUNAY Franck

LAVIEILLE Jean-Pierre

LE CORROLLER Thomas

LECHEVALLIER Eric

LEGRE Régis

LEHUCHER-MICHEL Marie-Pascale

LEONE Marc

LEONETTI Georges

LEPIDI Hubert

LEVY Nicolas

MACE LoÏc

MAGNAN Pierre-Edouard

MANCINI Julien

MEGE Jean-Louis

MERROT Thierry

METZLER/GUILLEMAIN Catherine

MEYER/DUTOUR Anne

MICCALEF/ROLL Joëlle

MICHEL Fabrice

MICHEL Gérard

MICHEL Justin

MICHELET Pierre

MILH Mathieu

MILLION Matthieu

MOAL Valérie

MORANGE Pierre-Emmanuel

MOULIN Guy

MOUTARDIER Vincent

NAUDIN Jean

NICOLAS DE LAMBALLERIE Xavier

NICOLLAS Richard

NGUYEN Karine

OLIVE Daniel

OLLIVIER Matthieu

OUAFIK L'Houcine

OVAERT-REGGIO Caroline

PADOVANI Laetitia 
TURRINI Olivier

VALERO René

VAROQUAUX Arthur Damien

VELLY Lionel

VEY Norbert

VIDAL Vincent

VIENS Patrice

VILLANI Patrick

VITON Jean-Michel

VITTON Véronique

VIEHWEGER Heide Elke détachement

VIVIER Eric

XERRI Luc 


\begin{tabular}{|c|c|}
\hline AHERFI Sarah & ELDIN Carole \\
\hline ANGELAKIS Emmanouil (disponibilité) & FAURE Alice \\
\hline ATLAN Catherine (disponibilité) & FOLETTI Jean- Marc \\
\hline BEGE Thierry & FRANKEL Diane \\
\hline BELIARD Sophie & FROMONOT Julien \\
\hline BENYAMINE Audrey & GASTALDI Marguerite \\
\hline BERTRAND Baptiste & GELSI/BOYER Véronique \\
\hline BEYER-BERJOT Laura & GIUSIANO Bernard \\
\hline BIRNBAUM David & GIUSIANO COURCAMBECK Sophie \\
\hline BONINI Francesca & GONZALEZ Jean-Michel \\
\hline BOUCRAUT Joseph & GOURIET Frédérique \\
\hline BOULAMERY Audrey & GRAILLON Thomas \\
\hline BOULLU/CIOCCA Sandrine & GUERIN Carole \\
\hline BOUSSEN Salah Michel & GUENOUN MEYSSIGNAC Daphné \\
\hline BUFFAT Christophe & GUIDON Catherine \\
\hline CAMILLERI Serge & GUIVARCH Jokthan \\
\hline CARRON Romain & HAUTIER/KRAHN Aurélie \\
\hline CASSAGNE Carole & HRAIECH Sami \\
\hline CERMOLACCE Michel & KASPI-PEZZOLI Elise \\
\hline CHAUDET Hervé & L'OLLIVIER Coralie \\
\hline CHRETIEN Anne-Sophie & LABIT-BOUVIER Corinne \\
\hline COZE Carole & LAFAGE/POCHITALOFF-HUVALE Marina \\
\hline CUNY Thomas & LAGARDE Stanislas \\
\hline DADOUN Frédéric (disponibilité) & LAGIER Aude (disponibilité) \\
\hline DALES Jean-Philippe & LAGOUANELLE/SIMEONI Marie-Claude \\
\hline DARIEL Anne & LEVY/MOZZICONACCI Annie \\
\hline DAUMAS Aurélie & LOOSVELD Marie \\
\hline DEGEORGES/VITTE Joëlle & MAAROUF Adil \\
\hline DELLIAUX Stéphane & MACAGNO Nicolas \\
\hline DESPLAT/JEGO Sophie & MAUES DE PAULA André \\
\hline DEVILLIER Raynier & MEGE Diane \\
\hline DUBOURG Grégory & MOTTOLA GHIGO Giovanna \\
\hline DUCONSEIL Pauline & NINOVE Laetitia \\
\hline DUFOUR Jean-Charles & NOUGAIREDE Antoine \\
\hline
\end{tabular}

PAULMYER/LACROIX Odile PESENTI Sébastien

RADULESCO Thomas

RESSEGUIER Noémie

ROBERT Philippe

ROBERT Thomas

ROMANET Pauline

SABATIER Renaud

SARI-MINODIER Irène

SAVEANU Alexandru

SECQ Véronique (disponibilité)

STELLMANN Jan-Patrick

SUCHON Pierre

TABOURET Emeline

TOGA Caroline

TOGA Isabelle

TOMASINI Pascale

TOSELLO Barthélémy

TROUSSE Delphine

TUCHTAN-TORRENTS Lucile

VELY Frédéric

VION-DURY Jean

ZATTARA/CANNONI Hélène

\title{
MAITRES DE CONFERENCES DES UNIVERSITES
}

(mono-appartenants)

ABU ZAINEH Mohammad
BARBACARU/PERLES T. A.
BERLAND Caroline
BOYER Sylvie
COLSON Sébastien
DEGIOANNI/SALLE Anna

DESNUES Benoît

MARANINCHI Marie

MERHEJ/CHAUVEAU Vicky

MINVIELLE/DEVICTOR Bénédicte

POGGI Marjorie

POUGET Benoît
RUEL Jérôme THOLLON Lionel

THIRION Sylvie

VERNA Emeline

MAITRE DE CONFERENCES DES UNIVERSITES DE MEDECINE GENERALE

CASANOVA Ludovic

MAITRES DE CONFERENCES ASSOCIES DE MEDECINE GENERALE à MI-TEMPS

\author{
BARGIER Jacques \\ CALVET-MONTREDON Céline \\ FORTE Jenny \\ JANCZEWSKI Aurélie \\ NUSSLI Nicolas \\ ROUSSEAU-DURAND Raphaëlle
}


THERY Didier

MAITRE DE CONFERENCES ASSOCIE à MI-TEMPS

BOURRIQUEN Maryline

EVANS-VIALLAT Catherine

LAZZAROTTO Sébastien

LUCAS Guillaume

MATHIEU Marion

MAYENS-RODRIGUES Sandrine

MELLINAS Marie

ROMAN Christophe

TRINQUET Laure 
CHAMPSAUR Pierre (PU-PH)

LE CORROLLER Thomas (PU-PH)

PIRRO Nicolas (PU-PH)

GUENOUN-MEYSSIGNAC Daphné (MCU-PH)

LAGIER Aude (MCU-PH) disponibilité

THOLLON Lionel (MCF) (60ème section)
ADALIAN Pascal (PR)

DEGIOANNI/SALLE Anna (MCF)

POUGET Benoît (MCF)

VERNA Emeline (MCF)

BACTERIOLOGIE-VIROLOGIE ; HYGIENE HOSPITALIERE 4501

CHARREL Rémi (PU PH)

DRANCOURT Michel (PU-PH)

FENOLLAR Florence (PU-PH)

FOURNIER Pierre-Edouard (PU-PH)

NICOLAS DE LAMBALLERIE Xavier (PU-PH)

LA SCOLA Bernard (PU-PH)

RAOULT Didier (PU-PH) Surnombre

CHARAFE/JAUFFRET Emmanuelle (PU-PH)

DANIEL Laurent (PU-PH)

FIGARELLA/BRANGER Dominique (PU-PH)

GARCIA Stéphane (PU-PH)

XERRI LUC (PU-PH)

AHERFI Sarah (MCU-PH)

ANGELAKIS Emmanouil (MCU-PH) disponibilité

DUBOURG Grégory (MCU-PH)

GOURIET Frédérique (MCU-PH)

NOUGAIREDE Antoine (MCU-PH)

DALES Jean-Philippe (MCU-PH)

NINOVE Laetitia (MCU-PH)

GIUSIANO COURCAMBECK Sophie (MCU PH)

LABIT/BOUVIER Corinne (MCU-PH)

CHABRIERE Eric (PR) (64ème section)

MACAGNO Nicolas (MCU-PH)

MAUES DE PAULA André (MCU-PH)

SECQ Véronique (MCU-PH) disponibilité
LEVASSEUR Anthony (PR) (64ème section)

DESNUES Benoit (MCF) ( 65ème section)

MERHEJ/CHAUVEAU Vicky (MCF) (87ème section)
ANESTHESIOLOGIE ET REANIMATION CHIRURGICALE ; MEDECINE URGENCE 4801

\author{
ALBANESE Jacques (PU-PH) \\ BRUDER Nicolas (PU-PH) \\ LEONE Marc (PU-PH) \\ MICHEL Fabrice (PU-PH) \\ VELLY Lionel (PU-PH)
}

BOUSSEN Salah Michel (MCU-PH)

GUIDON Catherine (MCU-PH)

\section{BIOCHIMIE ET BIOLOGIE MOLECULAIRE 4401}

BARLIER/SETTI Anne (PU-PH)

GABERT Jean (PU-PH)

GUIEU Régis (PU-PH)

OUAFIK L'Houcine (PU-PH)

BUFFAT Christophe (MCU-PH)

FROMONOT Julien (MCU-PH)

MOTTOLA GHIGO Giovanna (MCU-PH)

ROMANET Pauline (MCU-PH)

SAVEANU Alexandru (MCU-PH)

\section{ANGLAIS 11}

BRANDENBURGER Chantal (PRCE) retraite mars 2021

FRAISSE-MANGIALOMINI Jeanne (PRCE)

BIOLOGIE ET MEDECINE DU DEVELOPPEMENT

ET DE LA REPRODUCTION ; GYNECOLOGIE MEDICALE 5405

METZLER/GUILLEMAIN Catherine (PU-PH)

PERRIN Jeanne (PU-PH)

\section{BIOLOGIE CELLULAIRE 4403}

ROLL Patrice (PU-PH)

FRANKEL Diane (MCU-PH)

GASTALDI Marguerite (MCU-PH)

KASPI-PEZZOLI Elise (MCU-PH)

LEVY-MOZZICONNACCI Annie (MCU-PH) 
BELIN Pascal (PR) (69ème section)

RANJEVA Jean-Philippe (PR) (69ème section)

CAMMILLERI Serge (MCU-PH)

VION-DURY Jean (MCU-PH)
DEHARO Jean-Claude (PU-PH)

FRANCESCHI Frédéric (PU-PH)

HABIB Gilbert (PU-PH)

PAGANELLI Franck (PU-PH)

THUNY Franck (PU-PH)

\section{BIOSTATISTIQUES, INFORMATIQUE MEDICALE} ET TECHNOLOGIES DE COMMUNICATION 4604
GAUDART Jean (PU-PH)

GIORGI Roch (PU-PH)

MANCINI Julien (PU-PH)

CHAUDET Hervé (MCU-PH)

DUFOUR Jean-Charles (MCU-PH)

GIUSIANO Bernard (MCU-PH)

ABU ZAINEH Mohammad (MCF) (5ème section)

BOYER Sylvie (MCF) (5ème section)
BERDAH Stéphane (PU-PH)

HARDWIGSEN Jean (PU-PH)

MOUTARDIER Vincent (PU-PH)

SEBAG Frédéric (PU-PH)

SIELEZNEFF Igor (PU-PH)

TURRINI Olivier (PU-PH)

BEGE Thierry (MCU-PH)

BEYER-BERJOT Laura (MCU-PH)

BIRNBAUM David (MCU-PH)

DUCONSEIL Pauline (MCU-PH)

GUERIN Carole (MCU PH)

MEGE Diane (MCU-PH)

CHIRURGIE ORTHOPEDIQUE ET TRAUMATOLOGIQUE 5002

ARGENSON Jean-Noël (PU-PH)

BLONDEL Benjamin (PU-PH)

FLECHER Xavier (PU PH)

OLLIVIER Matthieu (PU-PH)

PARRATTE Sébastien (PU-PH) Disponibilité

ROCHWERGER Richard (PU-PH)

TROPIANO Patrick (PU-PH)

\section{CHIRURGIE INFANTILE 5402CHIRURGIE INFANTILE 5402}

GUYS Jean-Michel (PU-PH) Surnombre

JOUVE Jean-Luc (PU-PH)

LAUNAY Franck (PU-PH)

MERROT Thierry (PU-PH)

VIEHWEGER Heide Elke (PU-PH) détachement

DARIEL Anne (MCU-PH)

FAURE Alice (MCU PH)

PESENTI Sébastien (MCU-PH)

FACIALE ET STOMATOLOGIE 5503CHIRURGIE MAXILLO-FACIALE E

CHOSSEGROS Cyrille (PU-PH)

GUYOT Laurent (PU-PH)

FOLETTI Jean-Marc (MCU-PH)

PADOVANI Laetitia (PH-PH)

SALAS Sébastien (PU-PH)

VIENS Patrice (PU-PH)

SABATIER Renaud (MCU-PH)

TABOURET Emeline (MCU-PH) 
COLLART Frédéric (PU-PH)

D'JOURNO Xavier (PU-PH) DODDOLI Christophe (PU-PH)

FOUILLOUX Virginie (PU-PH)

GARIBOLDI Vlad (PU-PH)

MACE LOÏC (PU-PH)

THOMAS Pascal (PU-PH)

TROUSSE Delphine (MCU-PH)
CASANOVA Dominique (PU-PH)

LEGRE Régis (PU-PH)

BERTRAND Baptiste (MCU-PH)

HAUTIER/KRAHN Aurélie (MCU-PH)

CHIRURGIE VASCULAIRE ; MEDECINE VASCULAIRE 5104

ALIMI Yves (PU-PH)

AMABILE Philippe (PU-PH)

GASTROENTEROLOGIE ; HEPATOLOGIE ; ADDICTOLOGIE 5201

BARTOLI Michel (PU-PH)

BOUFI Mourad (PU-PH)

MAGNAN Pierre-Edouard (PU-PH)

BARTHET Marc (PU-PH)

PIQUET Philippe (PU-PH)

DAHAN-ALCARAZ Laetitia (PU-PH)

SARLON-BARTOLI Gabrielle (PU PH)

GEROLAMI-SANTANDREA René (PU-PH)

GRANDVAL Philippe (PU-PH)

HISTOLOGIE, EMBRYOLOGIE ET CYTOGENETIQUE 4202

LEPIDI Hubert (PU-PH)

BERBIS Philippe (PU-PH)

DELAPORTE Emmanuel (PU-PH)

GAUDY/MARQUESTE Caroline (PU-PH)

GROB Jean-Jacques (PU-PH)

RICHARD/LALLEMAND Marie-Aleth (PU-PH)
BEROUD Christophe (PU-PH)

KRAHN Martin (PU-PH)

LEVY Nicolas (PU-PH)

NGYUEN Karine (PU-PH)

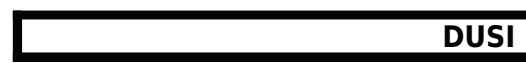

COLSON Sébastien (MCF)
TOGA Caroline (MCU-PH)

ZATTARA/CANNONI Hélène (MCU-PH)
BOURRIQUEN Maryline (MAST)

EVANS-VIALLAT Catherine (MAST)

LUCAS Guillaume (MAST)

MAYEN-RODRIGUES Sandrine (MAST)

MELLINAS Marie (MAST)

ROMAN Christophe (MAST)

TRINQUET Laure (MAST)
GYNECOLOGIE-OBSTETRIQUE ; GYNECOLOGIE MEDICALE 5403

AGOSTINI Aubert (PU-PH)

BOUBLI Léon (PU-PH) Surnombre

BRETELLE Florence (PU-PH)

CARCOPINO-TUSOLI Xavier (PU-PH)

COURBIERE Blandine (PU-PH)

ENDOCRINOLOGIE ,DIABETE ET MALADIES METABOLIQUES ;

CRAVELLO Ludovic (PU-PH)

D'ERCOLE Claude (PU-PH)

BRUE Thierry (PU-PH)

CASTINETTI Frédéric (PU-PH)

CUNY Thomas (MCU PH) 
AUQUIER Pascal (PU-PH)

BERBIS Julie (PU-PH)

BOYER Laurent (PU-PH)

GENTILE Stéphanie (PU-PH)

LAGOUANELLE/SIMEONI Marie-Claude (MCU-PH)

RESSEGUIER Noémie (MCU-PH)

MINVIELLE/DEVICTOR Bénédicte (MCF)(06ème section)
BLAISE Didier (PU-PH)

COSTELLO Régis (PU-PH)

CHIARONI Jacques (PU-PH)

GILBERT/ALESSI Marie-Christine (PU-PH)

MORANGE Pierre-Emmanuel (PU-PH)

VEY Norbert (PU-PH)

DEVILLIER Raynier (MCU PH)

GELSI/BOYER Véronique (MCU-PH)

LAFAGE/POCHITALOFF-HUVALE Marina (MCU-PH)

LOOSVELD Marie (MCU-PH)

SUCHON Pierre (MCU-PH)

POGGI Marjorie (MCF) (64ème section)

\section{MEDECINE LEGALE ET DROIT DE LA SANTE 4603}

KAPLANSKI Gilles (PU-PH)

MEGE Jean-Louis (PU-PH)

BARTOLI Christophe (PU-PH)

LEONETTI Georges (PU-PH)

PELISSIER-ALICOT Anne-Laure (PU-PH)

PIERCECCHI-MARTI Marie-Dominique (PU-PH)

FERON François (PR) (69ème section)

BOUCRAUT Joseph (MCU-PH)

TUCHTAN-TORRENTS Lucile (MCU-PH)

BERLAND Caroline (MCF) (1ère section)

MEDECINE PHYSIQUE ET DE READAPTATION 4905

MALADIES INFECTIEUSES ; MALADIES TROPICALES 4503

BROUQUI Philippe (PU-PH)

LAGIER Jean-Christophe (PU-PH)

MILLION Matthieu (PU-PH)

PAROLA Philippe (PU-PH)

STEIN Andréas (PU-PH)
MEDECINE ET SANTE AU TRAVAIL 4602

LEHUCHER/MICHEL Marie-Pascale (PU-PH)

ELDIN Carole (MCU-PH)

\section{MEDECINE D'URGENCE 4805}

KERBAUL François (PU-PH) détachement

MICHELET Pierre (PU-PH)

MEDECINE INTERNE ; GERIATRIE ET BIOLOGIE DU VIEILLISSEMENT ; ADDICTOLOGIE 5301

BONIN/GUILLAUME Sylvie (PU-PH)

DISDIER Patrick (PU-PH)

DURAND Jean-Marc (PU-PH)

EBBO Mikael (PU-PH)

GRANEL/REY Brigitte (PU-PH)

HARLE Jean-Robert (PU-PH)

ROSSI Pascal (PU-PH)

SCHLEINITZ Nicolas (PU-PH)

BENYAMINE Audrey (MCU-PH) 
GENTILE Gaëtan (PR Méd. Gén. Temps plein)

BRUNET Philippe (PU-PH)

BURTEY Stépahne (PU-PH)

CASANOVA Ludovic (MCF Méd. Gén. Temps plein)

DUSSOL Bertrand (PU-PH)

JOURDE CHICHE Noémie (PU PH)

GUIDA Pierre (PR associé Méd. Gén. à mi-temps) retraite au 25/09/2(MOAL Valérie (PU-PH)

BARGIER Jacques (MCF associé Méd. Gén. À mi-temps)

ROBERT Thomas (MCU-PH)

CALVET-MONTREDON Céline (MCF associé Méd. Gén. à temps plein)

FORTE Jenny (MCF associé Méd. Gén. À mi-temps)

JANCZEWSKI Aurélie (MCF associé Méd. Gén. À mi-temps)

NUSSLI Nicolas (MCF associé Méd. Gén. À mi-temps)

NEUROCHIRURGIE 4902

ROUSSEAU-DURAND Raphaëlle (MCF associé Méd. Gén. À mi-temps) DUFOUR Henry (PU-PH)

THERY Didier (MCF associé Méd. Gén. À mi-temps) (nomination au 1,FUENTES Stéphane (PU-PH)

REGIS Jean (PU-PH)

NUTRITION 4404

ROCHE Pierre-Hugues (PU-PH)

SCAVARDA Didier (PU-PH)

DARMON Patrice (PU-PH)

RACCAH Denis (PU-PH)

CARRON Romain (MCU PH)

VALERO René (PU-PH)

GRAILLON Thomas (MCU PH)

ATLAN Catherine (MCU-PH) disponibilité

BELIARD Sophie (MCU-PH)

MARANINCHI Marie (MCF) (66ème section)

\section{ONCOLOGIE 65 (BIOLOGIE CELLULAIRE)}

CHABANNON Christian (PR) (66ème section)

SOBOL Hagay (PR) (65ème section)

\section{OPHTALMOLOGIE 5502}

DAVID Thierry (PU-PH)

DENIS Danièle (PU-PH)

\section{NEUROLOGIE 4901}

ATTARIAN Sharham (PU PH)

AUDOIN Bertrand (PU-PH)

AZULAY Jean-Philippe (PU-PH)

CECCALDI Mathieu (PU-PH)

EUSEBIO Alexandre (PU-PH)

FELICIAN Olivier (PU-PH)

PELLETIER Jean (PU-PH)

MAAROUF Adil (MCU-PH)

\section{PEDOPSYCHIATRIE; ADDICTOLOGIE 4904}

DA FONSECA David (PU-PH)

POINSO François (PU-PH)

\section{OTO-RHINO-LARYNGOLOGIE 5501}

DESSI Patrick (PU-PH)

FAKHRY Nicolas (PU-PH)

GUIVARCH Jokthan (MCU-PH)

GIOVANNI Antoine (PU-PH)

LAVIEILLE Jean-Pierre (PU-PH)

PHARMACOLOGIE FONDAMENTALE -

PHARMACOLOGIE CLINIQUE; ADDICTOLOGIE 4803

MICHEL Justin (PU-PH)

NICOLLAS Richard (PU-PH)

TRIGLIA Jean-Michel (PU-PH)

BLIN Olivier (PU-PH)

MICALLEF/ROLL Joëlle (PU-PH)

SIMON Nicolas (PU-PH)

RADULESCO Thomas (MCU-PH)

REVIS Joana (PAST) (Orthophonie) (7ème Section)
BOULAMERY Audrey (MCU-PH) 
ANDRE Nicolas (PU-PH)

BARLOGIS Vincent (PU-PH)

CHAMBOST Hervé (PU-PH)

DUBUS Jean-Christophe (PU-PH)

FABRE Alexandre (PU-PH)

GIRAUD/CHABROL Brigitte (PU-PH)

MICHEL Gérard (PU-PH)

MILH Mathieu (PU-PH)

OVAERT-REGGIO Caroline (PU-PH)

REYNAUD Rachel (PU-PH)

TSIMARATOS Michel (PU-PH)

TOSELLO Barthélémy (MCU-PH)
BARTOLOMEI Fabrice (PU-PH)

BREGEON Fabienne (PU-PH)

GABORIT Bénédicte (PU-PH)

MEYER/DUTOUR Anne (PU-PH)

TREBUCHON/DA FONSECA Agnès (PU-PH)

BONINI Francesca (MCU-PH)

BOULLU/CIOCCA Sandrine (MCU-PH)

DADOUN Frédéric (MCU-PH) (disponibilité)

DELLIAUX Stéphane (MCU-PH)

LAGARDE Stanislas (MCU-PH)

RUEL Jérôme (MCF) (69ème section)

THIRION Sylvie (MCF) (66ème section)

\section{PSYCHIATRIE D'ADULTES ; ADDICTOLOGIE 4903}

\section{BAILLY Daniel (PU-PH)}

LANCON Christophe (PU-PH)

NAUDIN Jean (PU-PH)

RICHIERI Raphaëlle (PU-PH)

CERMOLACCE Michel (MCU-PH)

\section{PNEUMOLOGIE; ADDICTOLOGIE 5101}

ASTOUL Philippe (PU-PH)

BARLESI Fabrice (PU-PH)

CHANEZ Pascal (PU-PH)

GREILLIER Laurent (PU PH)

REYNAUD/GAUBERT Martine (PU-PH)

CHOLOGIE - PSYCHOLOGIE CLINIQUE, PCYCHOLOGIE SOCIALE 16

AGHABABIAN Valérie (PR)

TOMASINI Pascale (MCU-PH)

LAZZAROTTO Sébastien (MAST)

\section{RADIOLOGIE ET IMAGERIE MEDICALE 4302}

BARTOLI Jean-Michel (PU-PH)

CHAGNAUD Christophe (PU-PH)

CHAUMOITRE Kathia (PU-PH)

GIRARD Nadine (PU-PH)

JACQUIER Alexis (PU-PH)

MOULIN GUy (PU-PH)

PANUEL Michel (PU-PH) surnombre

PETIT Philippe (PU-PH)

VAROQUAUX Arthur Damien (PU-PH)

VIDAL Vincent (PU-PH)

STELLMANN Jan-Patrick (MCU-PH)

\section{RHUMATOLOGIE 5001}

GUIS Sandrine (PU-PH)

LAFFORGUE Pierre (PU-PH)

PHAM Thao (PU-PH)

ROUDIER Jean (PU-PH)

'HERAPEUTIQUE; MEDECINE D'URGENCE; ADDICTOLOGIE 480.

AMBROSI Pierre (PU-PH)

VILLANI Patrick (PU-PH)

DAUMAS Aurélie (MCU-PH) 
GAINNIER Marc (PU-PH)

GERBEAUX Patrick (PU-PH)

PAPAZIAN Laurent (PU-PH)

$\mathrm{ROCH}$ Antoine (PU-PH)

HRAIECH Sami (MCU-PH)
BASTIDE Cyrille (PU-PH)

KARSENTY Gilles (PU-PH)

LECHEVALLIER Eric (PU-PH)

ROSSI Dominique (PU-PH) 


\section{Remerciements :}

Je commence par remercier grandement le Docteur Benjamin COIFFARD, mon directeur de thèse, qui a eu l'idée de ce projet et sans qui ce travail n'aurait jamais vu le jour. J'ai apprécié créer de toutes pièces ce projet qui nous a fait nous creuser la tête pas mal de fois. Merci pour ton intérêt pédagogique et ton investissement personnel dans les projets dans lesquels tu t'engages. Tu as su éveiller en moi de l'intérêt et de la curiosité pour ce travail, me motiver dans mes moments de pessimisme et tu m'as dit les choses telles que tu les penses toujours dans le but de me faire progresser. Et en ce sens, tu m'as enseigné, alors merci.

Je remercie Madame la Professeure Martine REYNAUD-GAUBERT, ma présidente de jury, qui nous a soutenu dans ce projet depuis le départ et qui a également participé activement à l'écriture de ce travail. Merci de m'avoir fait l'honneur de présider mon jury.

Merci au Docteur Paul HABERT pour sa motivation, son efficacité et pour l'apport de son point de vue radiologique dans l'écriture de ce travail.

Merci également aux Docteur Ugo SCEMMAMA, au Docteur Basile PUECH et à Monsieur le Professeur Jean-Yves GAUBERT, pour leur aide et leur expertise radiologique nécessaires à l'élaboration de ce projet.

Merci à Zhonglin LI à Québec, qui nous a aidé à sortir d'une impasse informatique et nous a permis de poursuivre ce travail.

Je remercie tous mes professeurs et enseignants, toutes les personnes qui ont contribué à ma formation, pour la transmission de leurs connaissances et de leur passion ainsi que pour leur investissement personnel. Merci d'avoir contribué au bon apprentissage de mon métier.

Je remercie particulièrement le Professeur Pascal CHANEZ, qui m'a soutenu dans tous mes projets durant ce $3^{\text {ème }}$ cycle, qui m'a ouvert des portes, dont celle de son laboratoire, et qui a su faire preuve d'humanité et de compréhension à mon égard. Je vous remercie d'être venu réveiller la chercheuse qui sommeillait en moi et de me permettre de trouver ma voie professionnelle.

Je tiens à remercier ma famille sans qui rien de tout ça n'existerait. Maman, Papa, Mamie, Papi, Laura, Aymeric, Fannette, Chanto, souvent, je prends conscience de la chance que j'ai de vous avoir, vous qui êtes là depuis mes débuts (sauf Aymeric qui a loupé 2 ans et demi !). Je ne vous remercie pas seulement parce que vous m'avez éduqué, permis de réaliser mes études (merci !), appris à conduire, défendu à l'école, joué à la Barbie ou initié au zouk. Je tiens à vous remercier parce que vous m'avez donné de l'amour. Vous avez été là quoi qu'il arrive, m'avez soutenu quelques soient mes projets et mes décisions, sans jamais me juger, et ça m'a donné de la force tout au long de mon parcours. Je vous remercie aussi car vous m'avez inculqué des valeurs, vous m'avez appris à être juste, à connaitre la valeur des choses, à partager et à savoir donner sans demander en retour, appris à ouvrir mon esprit à la philosophie (cf les toilettes) et la spiritualité (dans un esprit de scientifique, ce n'était pas gagné !).

Tout cet amour, cet équilibre, cette reconnaissance m'ont aidé à me construire 
personnellement et professionnellement. La personne et le jeune médecin que je suis aujourd'hui, c'est en grande partie grâce à vous. Merci d'être là et de l'avoir toujours été. Je remercie aussi Papi Marcel et Mamie Augusta, que je n'ai pas eu la chance de découvrir comme je l'aurais voulu, mais qui m'ont probablement transmis via leur ADN les notions de rigueur, de travail et de don de soi.

Ma Fannette, Loïc, Sister cerise que j'aime, Maxime, mon petit frère adoré, petite Jen pleine d'amour : merci d'avoir régulièrement créé des rayons de soleil pour mon plus grand bonheur : notre Lulu pleine d'amour et de douceur, ma Nana coquine que j'aime tant, Louise, notre petit clown adoré, Aleyna notre rayon de soleil et bientôt...Joanne ! Vous me donnez foi en l'humanité et en la vie.

Xavier...j'avais prévu de te remercier mais j'ai ensuite réfléchi que tu envisageais de mettre une affiche de moi devant l'hôpital à mes 30 ans ....alors j'hésite... ! Je te remercie d'avoir été là quoi qu'il arrive depuis notre enfance, d'avoir supporté mes variations d'humeur (hum hum), d'avoir su me faire rire même dans les moments les plus durs ou stressants et surtout d'avoir su me rappeler pourquoi j'avais choisi ce métier lorsque j'en avais besoin. Ton courage et ton sens du travail forcent toujours mon admiration et me stimulent.

Ben, je pense que tu es le plus courageux dans cette histoire...merci de m'avoir accompagné, de m'avoir prêté ton bureau (qui m'a permis d'écrire cette thèse) et surtout de m'avoir supporté ces dernières semaines ce qui n'aurait pas été possible sans ta patience infaillible... merci d'être à mes côtés tout simplement.

Un grand merci à mon parrain Domi et ma marraine Marie-Jo, merci pour votre soutien sans faille, pour les petits plats apportés pendant que je travaille, pour votre disponibilité et vos attentions quotidiennes. Domi, tu m'as appris que l'on pouvait être un médecin ou un chirurgien brillant et être simple, honnête et modeste en même temps, et que mettre ses compétences au service des gens sans rien attendre en retour est le fondement de notre travail de «soignant». Merci pour ces leçons de vie.

Je tiens ensuite à remercier ma «famille professionnelle », avec qui j'ai passé ces dernières années plus de temps qu'avec ma propre famille.

Je remercie tous mes co-internes de pneumologie. On s'est souvent fait la réflexion avec plusieurs d'entre vous que l'internat (ou études du $3^{\text {ème }}$ cycle) de pneumologie est particulier en ce sens que l'on est plus soudés, qu'on porte attention les uns aux autres et que l'on se considère plus comme une famille réunie par sa passion pour la pneumologie que comme des êtres individuels. C'est exactement ce que j'ai ressenti avec vous, et je pense que ces années n'auraient pas été aussi agréables si vous n'aviez pas travaillé (et décompressé) à mes côtés.

Claire, Julie, Vincent (et son chat), Matthias, Ghada, Cécile (frites le mercredi !!), Bertrand (Pr Chollet), Alice (et notre bon vieux Roger...), Laura, Juju (l'espagnol), Fanny (l'insulaire), Matthieu, Solène, Chloé (super maman), Faridouuu, Thibaut, Alex6, Quentin(Balachoif), Eléonore (le fameux Simon), Julie(Kimanouuu), Laurent(et ses furettes), Mouloud : c'était un plaisir de « servir à vos côtés ». Ces stages n'auraient pas été les mêmes sans vous, merci de les avoir rendus si agréables. 
Un remerciement spécial pour Julien (alias Juju el Profesor Bermudez), qui a su me transmettre sa passion et sa motivation sans faille et qui m'a poussé (sans réellement le savoir) vers la voie de la recherche ; pour Alice et Laura, mes deux acolytes de vin blanc et bébés médecins devenues brillantes : c'est bon de vous avoir comme amies...; pour la team Kimanou ( Kimanou, Kimanou !) sans qui cette thèse n'existerait pas : merci de m'avoir donné du temps, et de m'avoir fait tant rire dans ces derniers mois pas toujours simples ; pour Solène, «l'autre fille de ma promo », ma compagnonne de chambre pendant tous ces séminaires: ta gentillesse n'a d'égal que ton professionnalisme, ne change jamais.

Merci aussi à Ju, Aurélien et Thibaut, mes 3 compagnons de route de la fac (les plus anciens !), mes sous-colleurs d'enfer, d'avoir partagé ces années d'études, ces heures de travail, ces beaux moments de rires et de pizzas ( l'idéale !) avec moi.

Merci à tous les québécois chers à mon cœur, qui m'ont accueilli chaleureusement et aux côtés desquels j'ai eu la chance de découvrir une autre façon de travailler et d'apprendre durant quelques mois. Un remerciement spécial pour la Professeure Jamila CHAKIR, le Docteur François MALTAIS et le Docteur Yohan BOSSÉ qui m'ont ouvert leurs portes et m'ont permis de m'enrichir professionnellement et culturellement. Merci pour votre gentillesse et vos attentions à mon égard.

Merci également à Luc et Laura, pour ces mois si agréables à vos côtés, à goûter tous les plats québécois. Merci de m'avoir initié au Rummikub québécois, qui, paraît-il, comporterait des pièces vertes.

Pour finir, je tiens à remercier la vie et l'Univers, car j'oublie souvent de les remercier.

Merci à vous de m'avoir mené sur ce chemin qui est le mien, de m'avoir permis de choisir cette famille, ce métier, d'avoir également rencontré toutes ces personnes qui à un moment donné sont montées dans le train de mon existence pour échanger, apprendre, évoluer, apporter ce qu'elles ont à m'apporter à leur façon et qui, même si elles n'y sont pas toutes restées, ont contribué à ma présence ici aujourd'hui et à ce travail, qui symbolise l'achèvement d'un bout de chemin et le début d'un autre. 
Rubrique «Articles originaux»

Densité pulmonaire et quantification vasculaire tomodensitométrique dans

l'hypertension pulmonaire associée aux pneumopathies interstitielles diffuses fibrosantes

Kim Valette ${ }^{1}$, Paul Habert ${ }^{2,3}$, Basile Puech ${ }^{2,3}$, Jean-Yves Gaubert ${ }^{2,3}$, Martine ReynaudGaubert $^{* 1}$, Benjamin Coiffard*1

1) Aix-Marseille Université, Service de Pneumologie, Assistance publique des hôpitaux de Marseille, Hôpital Nord, Chemin des Bourrely, 13915, Marseille

2) Aix-Marseille Université, Service de Radiologie, Assistance publique des hôpitaux de Marseille, Hôpital Nord, Chemin des Bourrely, 13915, Marseille

3) Aix-Marseille Université, Laboratoire d'Imagerie Interventionnelle Expérimentale (LIIE), Marseille, France

Titre court : Fibrose, densité et hypertension pulmonaire

Contribution des auteurs : KV : a,b,c,d; BP : a,d; JYG : a,b,d; PH : c,d; MRG : a,b,c,d; BC : $\mathrm{a}, \mathrm{b}, \mathrm{c}, \mathrm{d}$.

*2 Derniers auteurs ayant le même engagement

Soutiens : Cette étude n'a bénéficié d'aucun soutien financier.

Conflits d'intérêt : Les auteurs n'ont aucun conflit d'intérêt.

\section{Auteur correspondant :}

Kim Valette

35 Avenue des Pins

13013 Marseille

Tel : 0628944149

Email : kimvalette@gmail.com (email secondaire : Kim.VALETTE@ap-hm.fr) 


\section{Résumé}

(199 mots)

Introduction : L'hypertension pulmonaire (HTP) associée aux pathologies respiratoires est encore mal comprise. L'objectif principal était d'évaluer la relation entre tomodensitométrie (TDM) pulmonaire et HTP chez des patients atteints de pneumopathie interstitielle diffuse d'évolution fibrosante (PF-ILD).

Méthodes: L'étude a porté sur des PF-ILD, ayant tous bénéficié d'une TDM et d'un cathétérisme cardiaque droit avant transplantation pulmonaire. Les TDM ont fait l'objet d'une analyse quantitative de la densité et de la vascularisation pulmonaire. La relation entre TDM et HTP a été étudiée.

Résultats : 57 patients ont été inclus. La PAPm était corrélée avec la masse pulmonaire $(\mathrm{r}=0,35 ; \mathrm{p}=0,02)$ et le volume pulmonaire $(\mathrm{r}=0,45 ; \mathrm{p}=0,003)$. Les patients avec HTP sévère avaient plus de voxels d'unité Hounsfield (UH) inférieure à $-856(+16 \% ; p=0,02)$, moins de voxels d'UH supérieure à $-700(-20 \%$; p=0,03), et un volume pulmonaire plus élevé $(+1,55 \mathrm{~L}$, p=0,005) comparés à ceux sans HTP. Aucune corrélation n'a été retrouvée entre vascularisation et HTP.

Conclusions: L'analyse TDM quantitative permet d'établir un lien entre densité parenchymateuse et la présence d'une HTP dans la PF-ILD. Les patients atteints avec HTP sévère ont des atteintes interstitielles moins marquées que les patients avec HTP modérée ou pas d'HTP.

Mots-clés : Pneumopathie interstitielle idiopathique; Fibrose pulmonaire; Hypertension pulmonaire ; Tomodensitométrie ; Raréfaction de la vascularisation 
Title: Computed tomography lung density and small vessels quantification in pulmonary hypertension associated with progressive fibrosing interstitial lung disease (PF-ILD)

\section{Summary}

\section{(168 words)}

Introduction: Pulmonary hypertension $(\mathrm{PH})$ associated with respiratory diseases is still poorly understood. The aim of this work was to investigate a link between computed tomography and $\mathrm{PH}$ in patients with progressive fibrosing interstitial lung disease (PF-ILD).

Methods: This study included 57 patients with PF-ILD, all of whom underwent right cardiac catheterization and chest computed tomography before lung transplantation. Computed tomography scans were analyzed quantitatively for density and pulmonary vascularity. The relationship between computed tomography features and $\mathrm{PH}$ has been investigated.

Results: mPAP was positively correlated with lung mass $(\mathrm{r}=0.35, \mathrm{p}=0.02)$ and lung volume $(\mathrm{r}=0.45, \mathrm{p}=0.003)$. Patients with severe $\mathrm{PH}$ had more voxels less than -856 Hounsfield Unit (HU) $(+16 \%, \mathrm{p}=0.02)$, fewer voxels greater than $-700 \mathrm{HU}(-20 \%, \mathrm{p}=0.03)$, and a higher lung volume $(+1.55 \mathrm{~L}, \mathrm{p}=0.005)$ compared to patients without $\mathrm{PH}$. No correlation was found between vascularization and HTP.

Conclusions: Quantitative computed tomography analysis establishes a link between lung density and PH in PF-ILD. Patients with severe PH had less severe fibrosis than patients with moderate or without $\mathrm{PH}$.

Keywords: Interstitial Pneumonia; Pulmonary Fibroses; Pulmonary hypertension; X-Ray Computed Tomography; Microvascular Rarefactions 


\section{Introduction}

Le concept de pneumopathie interstitielle diffuse (PID) «d'évolution fibrosante» (progressive fibrosing interstitial lung disease (PF-ILD)), a été clairement défini récemment comme l'apparition, au cours du suivi d'une PID, d'un pattern tomodensitométrique de fibrose associé à une dégradation clinique et fonctionnelle respiratoire ainsi que de la qualité de vie, à une moindre réponse aux thérapies immunomodulatrices, et potentiellement une mortalité précoce [1]. Le modèle de référence actuel pour la prise en charge des PF-ILD est la fibrose pulmonaire idiopathique (FPI), la plus commune, et la plus largement étudiée des PID.

Parmi les co-morbidités associées aux PID, l'apparition d'une hypertension pulmonaire (HTP) va classiquement venir grever le pronostic [2, 3]. L'incidence de l'HTP dans ce contexte est très variable, mal précisée dans la plupart des études, probablement sous-estimée, allant de 32 à $85 \%$ chez les patients atteints de FPI [4] mais possiblement serait moindre $(<15 \%)$ pour les autres types de PID, en dehors de la sclérodermie.

Comme déjà démontré dans l'emphysème, il est désormais admis qu'il existe une dysrégulation de l'angiogenèse à l'origine d'anomalies de la vascularisation pulmonaire chez les patients atteints de PID [5]. Le type d'atteinte vasculaire est toutefois discordant dans la littérature, allant de l'hypovascularisation à l'hypervascularisation ou encore à une hétérogénéité spatiale et temporelle de la vascularisation pulmonaire selon les études, nécessitant une clarification. La dysrégulation de la vascularisation constitue un argument de poids pour expliquer le développement d'une HTP. Cependant, les raisons pour lesquelles tous les patients atteints de PID ne vont pas systématiquement développer une HTP, et celles pour lesquelles la sévérité de l'HTP est variable d'un individu à l'autre restent à ce jour inconnues. 
Pour l'heure, il n'existe aucun marqueur ni outil validé permettant de prédire la survenue d'une HTP chez ces patients.

La tomodensitométrie thoracique haute résolution (TDM-HR) constitue la pierre angulaire du diagnostic des PID, permettant de définir le pattern radiologique dans un but de classification diagnostique et pré-thérapeutique. L'intérêt pronostique de la TDM-HR a été récemment souligné. Ainsi, plusieurs études ont montré que des techniques d'analyse semiquantitative visuelle à partir d'un score de fibrose (estimant la quantité de réticulations et de rayon de miel) ou d'analyse quantitative de densité pulmonaire en 2D (à partir d'un histogramme de fréquence de pixels par unité Hounsfield (UH)) étaient corrélées à la fonction respiratoire $[6,7,8]$ et permettaient de prédire la progression de la maladie et la mortalité $[9$, $10,11]$.

La recherche de précision dans l'exploitation des données de la TDM thoracique a conduit au développement de techniques d'analyse en 3D désormais en plein essor. Ces nouveaux outils permettent, in vivo et in silico, d'être au plus près de la réalité anatomique de façon non invasive, laquelle était initialement uniquement accessible par l'anatomopathologie (biopsies pulmonaires). Ces outils d'analyse s'avèrent particulièrement intéressants dans des pathologies pulmonaires d'évolution fibrosante, où l'hétérogénéité et le caractère destructeur de la maladie rendent l'analyse histologique difficile, souvent peu informative et limitée au territoire analysé, reflétant mal de degré d'atteinte parenchymateuse globale.

Plusieurs études récentes suggèrent que, chez les patients atteints de PF-ILD, l'analyse quantitative de l'atteinte interstitielle et des vaisseaux pulmonaires en TDM utilisant des logiciels d'analyse 3D de densité et /ou de texture tels que l'outil CALIPER développé par la Mayo Clinic de Rochester (Minnesota) serait tout aussi, voire plus efficace, en termes de reflet de la fonction respiratoire, de prédiction de dégradation de la fonction respiratoire et de mortalité, que l'analyse semi-quantitative visuelle [12-16]. 
Matsuoka et coll., sur la base de troubles de la vascularisation pulmonaire décrits chez les patients atteints de bronchopneumopathie chronique obstructive (BPCO) avec emphysème, se sont intéressés à l'étude morphologique de la vascularisation périphérique chez ces patients [17]. Ils ont ainsi montré, par une technique de quantification vasculaire 2D basée sur la densité et la circularité des vaisseaux, que le pourcentage de vaisseaux de diamètre inférieur à $5 \mathrm{~mm}^{2}$ rapporté à la surface pulmonaire totale $\left(\% \mathrm{CSA}<5=\right.$ cross sectional area $\left.<5 \mathrm{~mm}^{2}\right)$ était significativement et très inversement corrélé à l'importance de l'emphysème $(r=-0,83)$. Ce résultat a été par la suite confirmé par une autre équipe, qui a associé deux notions importantes, que sont la précocité de l'apparition de l'hypovascularisation alors que l'emphysème était encore léger et sa proportionnalité par rapport à la sévérité de l'emphysème [18]. Ces différents travaux rapportaient également une corrélation inverse entre les vaisseaux de diamètre compris entre 5 et $10 \mathrm{~mm}^{2}$ et l'emphysème, mais de façon moins marquée et non ou peu significative.

Outre ce lien entre les vaisseaux de petit calibre et l'emphysème, le \%CSA $<5$ semble également être un outil prédictif de l'HTP (pression artérielle pulmonaire moyenne (PAPm)) : chez les patients atteints d'emphysème sévère, il est inversement corrélé à la PAPm de façon modérée et apparait comme un élément indépendant prédictif de la PAPm [19]. Chez les patients atteints d'HTP toutes causes confondues, hors BPCO, le \%CSA<5 était plus élevé dans le groupe atteint d'HTP que chez les contrôles (sujets sains sans HTP) mais au sein du groupe HTP, apparaissait la même corrélation inverse entre \%CSA<5 et PAPm [20].

Considérant ces données, nous émettions l'hypothèse qu'il pourrait, de la même façon que chez les patients atteints d'emphysème, exister un lien entre l'atteinte interstitielle parenchymateuse fibrosante, la présence et la sévérité d'une l'HTP ainsi qu'entre la vascularisation périphérique de petite taille et l'HTP chez les patients atteints de PF-ILD.

A ce jour, aucune corrélation n'a été mise en évidence entre densité pulmonaire par analyse 3D et HTP dans le cours évolutif des PID fibrosantes. Deux études seulement se sont 
intéressées à cette association : l'une concernant des patients atteints de FPI, l'autre des patients atteints de PID plus largement. Les résultats de ces deux études, qui reposaient sur une technique d'analyse semi-quantitative visuelle, ont été négatifs [21, 22]. Aucune étude ne s'est intéressée spécifiquement au lien potentiel entre la vascularisation de petit calibre en tomodensitométrie et la survenue d'une HTP chez les patients atteints de PID évolutive.

Notre travail avait donc deux objectifs : d'une part évaluer la corrélation entre HTP et analyse quantitative 3D de la densité pulmonaire ; et d'autre part, étudier le lien entre HTP et vascularisation périphérique dans une cohorte de patients atteints de PF-ILD.

\section{Méthodes}

\section{Population de l'étude}

Il s'agit d'une étude rétrospective, qui a inclus des patients âgés de plus de 18 ans, pour lesquels un diagnostic de PID d'évolution fibrosante avait été posé en réunion de discussion multidisciplinaire (DMD) selon l'anamnèse, le tableau clinique et radiologique, et ayant secondairement bénéficié d'une transplantation pulmonaire au CHU Nord de Marseille entre Janvier 2011 et Décembre 2019. Seuls les tableaux de pneumopathie interstitielle commune (PIC) histologiquement définis sur les poumons explantés ont été retenus. Les patients atteints de sclérodermie systémique étaient exclus.

Tous les patients devaient avoir bénéficié d'une tomodensitométrie thoracique et d'un cathétérisme cardiaque droit dans le cadre des explorations avant la transplantation.

Les données cliniques suivantes étaient recueillies : date de naissance, sexe, taille, poids, indice de masse corporelle (IMC), date de transplantation, traitements spécifiques anti- 
fibrosants, à visée cardio-vasculaire, et spécifiques de l'HTP (si utilisés) avant transplantation, oxygénothérapie et débit maximal avant transplantation.

\section{Éthique}

Cette étude a reçu l'aval du comité d'éthique de la Société de Pneumologie de Langue Française (SPLF) (CEPRO 2020-027) et l'autorisation du Délégué à la Protection des Données de l'AP-HM (DPO) pour le recueil et l'exploitation des données (RGPD/AP-HM 2020-60).

\section{Données fonctionnelles respiratoires}

Les données suivantes de l'épreuve fonctionnelle respiratoire (EFR) la plus proche en termes de date de la tomodensitométrie thoracique sélectionnée pour l'analyse était recueillies pour chaque patient: capacité vitale forcée (CVF), volume expiratoire maximal seconde (VEMS), capacité pulmonaire totale (CPT), capacité de diffusion du CO (DLCO), coefficient de diffusion du CO (DLCO/VA). Les données des gaz du sang et du test de marche de 6 minutes (TM6) étaient également recueillies.

\section{Données hémodynamiques (Cathétérisme cardiaque droit)}

Les cathétérismes cardiaques droits avaient tous été réalisés au sein du service de cardiologie du CHU Nord de Marseille (AP-HM), par les mêmes cardiologues expérimentés. Les données recueillies pour cette étude étaient les suivantes : PAPm, pression artérielle pulmonaire systolique (PAPs), pression artérielle pulmonaire diastolique (PAPd), pression capillaire (Pcap), débit cardiaque (DC), index cardiaque (IC), résistances vasculaires pulmonaires (RVP). Nous avions sélectionné les derniers cathétérismes réalisés avant la 
transplantation, et en dehors de toute exacerbation de PF-ILD ou d'évènement aigu. Les données de l'échocardiographie transthoracique la plus proche du cathétérisme étaient également recueillies, ainsi que le dosage sanguin du brain natriuretic peptide (BNP).

\section{Définition de l'hypertension pulmonaire}

L'HTP était définie dans cette étude selon la définition en vigueur lors de la réalisation des cathétérismes cardiaques droit, à savoir une HTP du groupe 3 pré-capillaire avec une PAPm $\geq 25 \mathrm{mmHg}$ et une pression capillaire (Pcap) $\leq 15 \mathrm{mmHg}$ sur une mesure effectuée au cours d'un cathétérisme cardiaque droit réalisé au repos en décubitus, à l'état de base [23].

Sur la base de cette définition, les patients étaient classés en 3 groupes selon les valeurs de la PAPm : «Pas d'HTP » lorsque la PAPm était inférieure à 25 mmHg, « HTP modérée » lorsque la PAPm était comprise entre 25 et 35 mmHg, « HTP sévère » lorsque la PAPm était supérieure à $35 \mathrm{mmHg}$.

\section{Analyse tomodensitométrique}

Les analyses ont porté sur les TDM thoraciques les plus proches de la date de réalisation du dernier bilan hémodynamique (cathétérisme cardiaque droit) avant la transplantation. Elles devaient avoir été réalisées en dehors de tout évènement respiratoire aigu. Le protocole d'acquisition était standardisé pour chaque TDM : toutes les acquisitions ont été réalisées de façon hélicoïdale, la tension du tube était appliquée au morphotype du patient (100kV pour les patients dont le poids était compris entre 60 et $100 \mathrm{~kg}, 80 \mathrm{kV}$ pour les patients dont le poids étaient inférieur à $60 \mathrm{~kg}$ et $120 \mathrm{kV}$ si le poids était supérieur à $100 \mathrm{~kg}$ ). Une modulation automatique des milliampères était réalisée, toutes les acquisitions ont été faites en inspiration 
maximale et en décubitus dorsal. Les données tomodensitométriques avaient ensuite fait l'objet de reconstructions en coupe de 1:1.

L'analyse densitométrique du parenchyme pulmonaire a été réalisée à l'aide d'un logiciel d'analyse 3D (3D Slicer, version 4.10.2 avec ajout de l'extension Chest Imaging Platform). Les séries avec un filtre dur parenchymateux ont été utilisées, la segmentation des régions pulmonaires a été réalisée à l'aide d'une méthode de seuillage en sélectionnant une fenêtre de voxels compris entre -1024 et -350 UH, à l'aide d'un module permettant d'exclure automatiquement les voies aériennes proximales afin qu'il ne s'agisse pas d'un facteur confondant dans la mesure. L'ensemble du volume final faisait l'objet d'une reconstruction en rendu de volume (Fig. 1). A partir de cette reconstruction, en se basant sur l'échelle des UH (Fig. 2), l'histogramme de quantification des voxels par UH était extrait. Le volume (L) et la masse (g) pulmonaire étaient calculés à partir des dimensions et la densité des deux poumons. La densité pulmonaire moyenne (MLD, moyenne des UH sur l'ensemble des voxels pulmonaires sélectionnés), les pourcentages de voxels de faible densité (\%LAA= low attenuation area) et de haute densité $(\% \mathrm{HAA}=$ high attenuation area) étaient calculés à partir des données de l'histogramme (Fig. 3). Les différents seuils considérés dans cette étude concernant le \%LAA étaient $\leq-950 \mathrm{UH},-925 \mathrm{UH},-910 \mathrm{UH},-856 \mathrm{UH}$ (correspondant globalement à l'air du parenchyme normal, ou au rayon de miel ou à de l'emphysème en cas de destruction), et pour le \%HAA $\geq-700 \mathrm{UH},-600 \mathrm{UH},-500 \mathrm{UH}$ et $-250 \mathrm{UH}$ (correspondant globalement aux réticulations, au verre dépoli et aux différentes structures tissulaires tels que les vaisseaux et les parois bronchiques principalement). Le coefficient d'aplatissement de la courbe (Kurtosis) et le coefficient d'asymétrie (Skewness) étaient déterminés à partir de la distribution de la densité pulmonaire représentée sur cet histogramme. Le Kurtosis représente le coefficient d'aplatissement : plus il est positif ou haut, plus le sommet de la courbe est pointu. Le Skewness représente le degré d'asymétrie de la courbe par rapport à une distribution 
normale, plus il se rapproche de 0 , plus la distribution des UH tend vers une distribution symétrique (normale). Le Skewness peut être positif ou négatif en fonction du côté de prédominance de l'asymétrie : il est positif en cas d'asymétrie prédominant à gauche et négatif en cas d'asymétrie prédominant à droite. Il a bien été décrit dans la littérature que lorsqu'il existe une atteinte fibrosante parenchymateuse, le Kurtosis et le Skewness diminuent, de telle sorte qu'il existe un aplatissement de la courbe et une diminution de l'asymétrie de répartition des UH [24]. Ces notions sont illustrées dans la Figure 4 qui compare les histogrammes d'un poumon normal à celui d'un patient atteint de PF-ILD.

La quantification vasculaire a été réalisée en 2D à l'aide du logiciel ImageJ (version 1.8.0 https://imagej.nih.gov/ij/). Les analyses ont été réalisées en utilisant les séries avec un filtre dur parenchymateux et aux 3 niveaux de coupe suivants : coupe « apicale » à $1 \mathrm{~cm}$ audessus de la crosse aortique, coupe «moyenne » à $1 \mathrm{~cm}$ en dessous de la carène, et coupe «basale » à $1 \mathrm{~cm}$ en dessous de la veine pulmonaire lobaire inférieure droite. Comme illustré dans la Figure 5, le parenchyme pulmonaire était sélectionné à l'aide d'un outil de segmentation automatique puis les autres structures étaient effacées. La surface obtenue avait de nouveau fait l'objet d'un seuillage, en supprimant tous les voxels de densité inférieure à - 600 UH (correspondant à la densité des vaisseaux), puis la sélection des vaisseaux était affinée sur la base de leur circularité (réglée entre 0,9 et 1). La surface des vaisseaux était ensuite extrapolée en fonction de leur diamètre puis classée en 3 catégories (de 0 à $5 \mathrm{~mm}^{2}$, de 5 à $10 \mathrm{~mm}^{2}$ et 10 $20 \mathrm{~mm}^{2}$ ) et rapportée à la surface pulmonaire totale de la coupe sélectionnée pour obtenir des $\%$ de surface de vaisseaux par rapport à la surface pulmonaire totale $(\% \mathrm{CSA}=$ cross sectional area).

\section{Statistiques}


Une analyse descriptive a été effectuée sur l'ensemble de la population et par groupe selon le niveau d'HTP (pas d'HTP, HTP modérée, HTP sévère). Les variables continues sont exprimées en médianes et interquartiles, ou moyennes et écarts-types selon la distribution (test de Shapiro-Wilk), et les variables qualitatives exprimées en nombres et en pourcentages.

Des analyses ont été réalisées pour comparer les caractéristiques entre les groupes. Les paramètres qualitatifs ont été comparés à l'aide des tests Chi-2. Les paramètres quantitatifs ont été comparés en utilisant un test de Student ou un test non paramétrique de Mann-WhitneyWilcoxon selon la distribution.

Des tests de corrélation entre les PAPm (en tant que variable numérique continue) et les variables de quantification tomodensitométriques (densité pulmonaire et vascularisation) ont été réalisés par la méthode de Pearson ou de Spearman selon la distribution. Les intervalles de confiances à 95\% de ces corrélations ont été calculés par méthode bootstrap à 1000 réplicas.

Les différences entre les groupes d'HTP pour les variables de quantification tomodensitométrique (densité pulmonaire et vascularisation) ont été étudiées par régression logistique avec le modèle linéaire général. Les coefficients de régression $\beta$ estiment la différence entre les groupes.

Tous les tests étaient bilatéraux. Une valeur $\mathrm{p}<0,05$ a été considérée comme significative. L'analyse a été effectuée en utilisant la version 3.5.1 (2018-07-02) du logiciel R (R Core Team (2018). R: A language and environment for statistical computing. R Foundation for Statistical Computing, Vienna, Austria. URL https://www.R-project.org/). 


\section{Résultats}

\section{Caractéristiques de la population}

L'étude a porté sur 57 patients atteints de PID fibrosantes. Parmi eux, 20 ne présentaient pas d'HTP (PAPm< 25 mmHg), 33 une HTP modérée (25-35 mmHg) et 4 une HTP sévère (> $35 \mathrm{mmHg}$ ). Les principales caractéristiques des patients étaient comparables dans les 3 groupes (Tableau 1) à l'exception : 1) de la proportion de femmes qui était d'autant plus faible que l'HTP était importante (63\% dans le groupe sans HTP, 37\% dans le groupe HTP non sévère, 0\% dans le groupe HTP sévère, $\mathrm{p}=0,02) ; 2$ ) des signes de surcharge cardiaque droite en ETT qui étaient d'autant plus présents que l'HTP était sévère; et 3) de la prise de traitements diurétiques qui étaient d'autant plus importante que l'HTP était sévère et de la prise de traitement antihypertenseur pulmonaire qui était propre au groupe HTP sévère.

Les PF-ILD étaient principalement représentées par les fibroses pulmonaires idiopathiques (42\%), suivies des PID en lien avec des connectivites (11\%) et des PID inclassables (11\%). Les autres étiologies de PF-ILD sont détaillées dans le Tableau 1. Ces étiologies étaient concordantes avec les principales catégories de PID à risque d'évolution fibrosante décrites dans la littérature [1].

\section{Densité pulmonaire et HTP}

La médiane de latence entre la réalisation du cathétérisme cardiaque droit et la tomodensitométrie était comparable dans les 3 groupes (pas d'HTP, HTP modérée, HTP sévère) et inférieure à 1 mois (Tableau 1). 
En termes d'analyse de densité, cette étude a mis en évidence une corrélation modérée significative des chiffres de PAPm $(\mathrm{mmHg})$ avec la masse totale en grammes $(r=0,35 ; \mathrm{p}=0,02)$ et le volume pulmonaire total en litres $(\mathrm{r}=0,45 ; \mathrm{p}=0,003)$ (Tableau 2).

La PAPm semblait également corrélée positivement aux différents seuils de \%LAA (\%LAA $\leq-950 \mathrm{UH},-925 \mathrm{UH},-910 \mathrm{UH},-856 \mathrm{UH}$ ), au Kurtosis et au Skewness, et corrélée négativement aux différents seuils de \%HAA ( $\geq-700 \mathrm{UH},-600 \mathrm{UH},-500 \mathrm{UH}$ et $-250 \mathrm{UH})$ et à la MLD, indiquant que la PAPm était d'autant plus élevée que la densité pulmonaire et notamment l'atteinte fibrosante était faible. Toutefois, ces corrélations étaient légères et non statistiquement significatives (Tableau 2).

Lorsque nous considérions 3 groupes de patients : 1) pas d'HTP, 2) HTP modérée, et 3 ) HTP sévère selon les définitions détaillées, il n'existait pas de différence significative concernant les différents paramètres d'analyse densitométrique entre les individus présentant une HTP modérée et ceux ne présentant pas d'HTP en régression linéaire. En revanche, par rapport aux individus sans HTP, les TDMs des individus avec HTP sévère contenaient significativement plus de \%LAA ( $\beta=6,71 \%$ à $16 \%, p=0,04$ à 0,02$)$, un volume pulmonaire total plus élevé $(\beta=1,55 \mathrm{~L}, \mathrm{p}=0,005)$ et un \%HAA (\%HAA $-700 \mathrm{UH}: \beta=-20,20 \%, p=0,03$; $\%$ HAA $-600-250 \mathrm{UH}: \beta=-11,40 \%, \mathrm{p}=0,04)$ plus faible. On pouvait également noter une tendance à une moindre MLD, sans que le résultat n'atteigne le seuil statistiquement significatif $(\beta=-98 \mathrm{UH}, \mathrm{p}=0,06)$.

Des résultats similaires étaient observés lorsqu'on comparait les individus avec une HTP sévère et ceux avec une HTP modérée. Les TDM des individus atteints d'HTP sévère montraient significativement plus de $\%$ LAA $(\beta=6,66 \%$ à $16,18 \%, p=0,01$ à 0,02$)$, un volume pulmonaire total plus élevé $(\beta=1,20 \mathrm{~L}, \mathrm{p}=0,03)$ et un \%HAA plus faible $(\% \mathrm{HAA}-700 \mathrm{UH}: \beta=-22 \%$, $\mathrm{p}=0,01 ; \%$ HAA $-600-250 \mathrm{UH}: \beta=-12 \%, \mathrm{p}=0,03)$ plus faible et une MLD plus faible $(\beta=-$ $116,81 \mathrm{UH}, \mathrm{p}=0,03)$. 
Les coefficients de Kurtosis et de Skewness semblaient plus élevés chez les individus atteints d'HTP sévère (Fig. 6) par rapport aux 2 autres groupes, sans significativité statistique.

\section{Vascularisation pulmonaire et HTP}

Nous n'avons pas trouvé de corrélation significative entre la vascularisation pulmonaire, quel que soit le diamètre vasculaire considéré $\left(<5 \mathrm{~mm}^{2}, 5-10 \mathrm{~mm}^{2}, 10-20 \mathrm{~mm}^{2}\right)$, et l'HTP. Il n'existait pas non plus de corrélation lorsqu'on considérait le gradient de vascularisation entre régions supérieures et inférieures. Les résultats représentés dans le Tableau 2 concernent uniquement la surface des vaisseaux $<5 \mathrm{~mm}^{2}(\mathrm{CSA}<5)$.

Fonction respiratoire, densité pulmonaire et vascularisation

L'estimation du volume pulmonaire total en tomodensitométrie était bien corrélée dans ce travail à la capacité pulmonaire totale mesurée lors d'une épreuve fonctionnelle respiratoire (Fig. 7).

Parmi les différents paramètres physiologiques respiratoires, il existait une corrélation inverse significative et modérée entre densité pulmonaire et CVF ainsi qu'entre densité pulmonaire et CPT (Tableau 3) : la CVF et la CPT étaient d'autant plus faible (indiquant une restriction importante) que la densité pulmonaire était élevée (\%HAA et MLD augmentés, Kurtosis et Skewness diminués) donc que l'atteinte fibrosante était importante. Il n'existait en revanche pas de corrélation entre densité pulmonaire et capacité de diffusion pulmonaire (DLCO) (Tableau 3). Aucune corrélation n'était observée lorsqu'on ajustait le DLCO au volume alvéolaire (KCO). 
Aucune corrélation significative n'a été mise en évidence entre vascularisation pulmonaire et fonction respiratoire.

\section{Discussion}

Cette étude rétrospective a permis de mettre en évidence un lien entre densité pulmonaire en tomodensitométrie et HTP chez les patients atteints de PF-ILD. Les principaux résultats apportés par ce travail concernent le groupe de patients atteints d'HTP sévère : leurs tomodensitométries montrent un \%LAA plus élevé, un volume pulmonaire total plus élevé et un moindre \%HAA, de manière significative. L'histogramme de répartition des densités des patients ayant une HTP sévère (Fig. 6) illustre bien ce pattern moins « fibrosé » que celui des patients avec HTP modérée ou sans HTP. Cependant, le pourcentage de voxels de faible densité tel que défini dans le logiciel utilisé dans ce travail regroupe les densités de -1024 à -856 UH, qui peuvent correspondre à la fois au parenchyme pulmonaire normal (estimé entre -950 et $856 \mathrm{UH}$ ) et aux lésions destructrices telles que le rayon de miel (estimées entre -1000 et -950 UH). Le pourcentage de voxels ayant une densité supérieure à -950 UH (\%LAA-950) est plus élevée chez les patients atteints d'HTP sévère que chez ceux avec HTP modérée ou sans HTP. Ce résultat est en faveur d'une plus grande proportion de lésions destructrices chez les patients ayant une HTP sévère. Toutefois, lorsque l'on compare visuellement l'aspect tomodensitométrique des patients ayant une HTP sévère par rapport à celui de patients ayant une HTP modérée ou n'ayant pas d'HTP, nous ne sommes pas tant impressionnés par la proportion de lésions en rayon de miel que par le fait qu'il semble surtout exister moins d'hyperdensités de type réticulations ou verre dépoli et plus de zones de poumon « normal » chez les individus atteints d'HTP sévère. Cet élément est reflété dans le Tableau 2 par le pourcentage de voxels de haute densité (\%HAA) > -700 et entre -600 et -250 UH qui sont significativement plus faibles chez les patients atteints d'HTP sévère que chez ceux avec HTP 
modérée $(\beta=-22 \%, p=0,01$ et $\beta=-12 \%, p=0,03$ respectivement $)$ ou sans $\operatorname{HTP}(\beta=-20 \%, p$ $=0,03$ et $\beta=-11 \%, p=0,04$ respectivement). De plus, le groupe de patients atteints d'HTP sévère a un volume pulmonaire total plus élevé que les autres groupes, ce qui, du fait de la bonne corrélation du volume avec la CPT (Fig. 7), rend compte d'une restriction moindre chez ces patients, témoignant d'une atteinte fibrosante moins avancée que dans les groupes avec HTP modérée ou sans HTP. Si cette donnée visuelle nécessite une confirmation objective quantitative en individualisant chaque type de lésion (verre dépoli, réticulations et rayon de miel), elle amène néanmoins à évoquer la notion d'une HTP « disproportionnée » par rapport à l'atteinte parenchymateuse dans le groupe d'individus atteints d'HTP sévère.

Ce concept a déjà été évoqué à plusieurs reprises dans la littérature. Nathan et al. [25] dans une étude portant sur 118 patients atteints de FPI, partant de l'hypothèse que la sévérité de la fibrose et l'importance de la restriction fonctionnelle étaient proportionnelles au degré de l'HTP, ont observé la situation inverse, avec une HTP plus sévère chez les patients ayant restriction moindre (CVF et $\mathrm{CPT}>70 \%$ th) comparés à ceux ayant une restriction plus sévère (CVF et CPT $<40 \%$ th). Une observation similaire a été rapportée dans un contexte de patients atteints de sarcoïdose et d'histiocytose langerhansienne [26, 27]. De la même manière, Hurdman et al. [28] dans une synthèse des caractéristiques des patients présentant une BPCO associée à une HTP inclus dans la cohorte ASPIRE (Assessing the Spectrum of Pulmonary Hypertension Identified at a Referral Centre), ont rapporté que les patients ayant une HTP sévère ne présentent pas plus de lésions d'emphysème sur leurs TDM que les patients ayant une HTP légère à modérée, et que leur trouble ventilatoire obstructif apparait moins important. L'analyse histologique d'explants montre que les artères et artérioles pulmonaires des individus atteints de BPCO avec HTP sévère, contrairement à ceux présentant une HTP non sévère, font l'objet d'un remodelage majeur avec des lésions intimales fibrosantes de haut grade, irréversibles, jamais décrites dans les HTP hypoxiques « habituelles » suggérant que les 
mécanismes sous-jacents de l'HTP sévère chez les patients atteints d'une pathologie respiratoire vont au-delà des remaniements et de la vasoconstriction hypoxique seule [29]. Ces résultats incitent à reconsidérer la position et la prise en charge ultérieure des HTP sévères du groupe 3 de la classification internationale [30] qui, quelle que soit la pathologie respiratoire associée, semblent constituer une entité à part entière. Des études complémentaires sur ce groupe 3 paraissent donc indispensables, en dépit de la faible population concernée, mais très probablement sous-estimée (rares données hémodynamiques disponibles chez ces patients en dehors d'un projet de transplantation pulmonaire, ou d'anomalies cardiaques droites préexistantes). Ainsi, la prévalence dans notre travail d'une HTP sévère, eu égard au stade très avancé d'insuffisance respiratoire restrictive, était seulement de $7 \%$, ce qui correspond à la prévalence d'HTP sévère associée aux PID retrouvée dans la littérature qui est d'environ 10\% $[31,32]$.

Ce travail met également en évidence une corrélation modérée significative entre le volume pulmonaire total et la PAPm ainsi qu'entre la masse pulmonaire et la PAPm. Ainsi, plus la PAPm est élevée (donc l'HTP est sévère) plus le volume pulmonaire et la masse sont élevés. De même, nous retrouvons une tendance à leur élévation dans les groupes présentant une HTP (modérée ou sévère) par rapport au groupe ne présentant pas d’HTP. Le volume pulmonaire estimé en TDM mérite cependant d'être interprété avec précaution du fait de la variabilité de l'inflation lors de l'acquisition en inspiration. Sa bonne corrélation avec la CPT dans cette étude (Fig. 7) est toutefois rassurante car elle semble refléter la capacité inspiratoire des patients avec une certaine reproductibilité lors de l'acquisition. La masse dépend de deux paramètres : le volume pulmonaire et la densité pulmonaire moyenne (masse = volume $\mathrm{x}$ densité). L'estimation de la masse est généralement moins artéfactée par les différences d'inspiration, étant basée sur la densité pulmonaire qui est elle-même basée sur le degré d'expansion (volume). Si l'on s'en tient aux résultats de corrélation entre la PAPm, la masse et le volume, constatant que la MLD 
n'est pas nettement corrélée à la PAPm, nous aurions tendance à conclure que l'augmentation de la masse est principalement en lien avec l'augmentation de volume. Cependant, lorsque nous analysons en détails les résultats par groupe d'HTP (Tableau 2), nous constatons qu'il existe une différence entre le groupe HTP sévère et le groupe HTP modérée : dans le groupe HTP sévère, la MLD est plus faible (MLD : $\beta=-98 \mathrm{UH}, \mathrm{p}=0,06$ ) et le volume significativement plus élevé (Volume : $\beta=1,5 \mathrm{~L}, \mathrm{p}=0,005$ ) que dans le groupe sans HTP. La tendance à l'augmentation de la masse chez les patients ayant une HTP sévère semble donc liée à l'augmentation de volume. En revanche, dans le groupe avec HTP modérée, la MLD tend à augmenter (MLD : $\beta$ $=+18,8 \mathrm{UH}, \mathrm{p}=0,45)$ alors que le volume n'est que faiblement augmenté, et ce de manière non significative (Volume : $\beta=0,34 \mathrm{~L}, \mathrm{p}=0,21$ ), par rapport au groupe sans HTP. Ceci pourrait suggérer que, chez les patients ayant une HTP modérée, l'augmentation de la masse est principalement en lien avec l'augmentation de la densité pulmonaire (MLD). Nous pouvons émettre ainsi plusieurs hypothèses pour expliquer une densité et donc une masse pulmonaire plus élevée chez les patients atteints d'HTP modérée par rapport à ceux ne présentant pas d'HTP. La première hypothèse serait l'existence d'une atteinte fibrosante parenchymateuse plus importante, proportionnelle à la PAPm. Cependant, les résultats sur la totalité de la cohorte, bien que non significatifs, ne vont pas dans ce sens. De plus, le \% HAA n'est que légèrement plus élevé dans ce groupe par rapport à celui sans HTP (\%HAA>-700 $\beta=1,80 \%, p=0,70$; $\%$ HAA-600-250 $\beta=0,59 \%, p=0,84)$, et le Kurtosis et le Skewness ne sont pas en faveur de cette hypothèse. Une autre hypothèse serait que l'élévation de la densité serait en lien avec des conséquences de 1'HTP à savoir un remodelage avec épaississement de la paroi vasculaire à l'origine d'une augmentation de la densité pulmonaire moyenne.

Toujours en considérant la PAPm comme variable linéaire, nous constatons dans cette étude, que la PAPm tend à être corrélée positivement au \%LAA, au Kurtosis, au Skewness et inversement au \%HAA et à la MLD, bien que ces corrélations soient légères et non 
significatives. Il apparait donc une tendance vers une densité pulmonaire d'autant plus faible et un aspect d'autant moins « fibrosant » que la PAPm est élevée. Ces résultats vont à l'encontre de l'hypothèse selon laquelle l'HTP serait secondaire et proportionnelle à l'atteinte interstitielle, et serait par conséquent d'autant plus élevée que la fibrose pulmonaire serait évoluée. En effet, deux études ayant évalué le lien potentiel entre atteinte interstitielle (verre dépoli, réticulations, rayon de miel) et PAPm, ont montré une relation positive dans ce sens, et donc des résultats contradictoires avec ceux que nous avons obtenus. Néanmoins, le faible niveau de corrélation n'a pas suffi à autoriser les auteurs à conclure sur une relation entre PAPm et atteinte interstitielle en TDM [21, 22]. De surcroît, nos algorithmes d'analyse tomodensitométrique n'étaient pas comparables. En effet, les deux études précédemment évoquées utilisaient des techniques d'analyse semi-quantitative visuelle qui consistaient à estimer visuellement le pourcentage de chaque type de lésions interstitielles aboutissant à un score, laissant la place à une possible subjectivité et à une variabilité inter-observateur dans l'analyse des images.

Par ailleurs, afin d'affiner nos résultats d'analyse de densité pulmonaire, il serait intéressant, par la suite, d'estimer la proportion de rayon de miel parmi les zones de faible densité. Plusieurs études ont mis au point des techniques dans cette optique, soit par analyse en 2D sur coupes tomodensitométriques à partir d'analyse de densités et de surfaces prédéfinies $[6,33]$, soit par repérage manuel de texture en 2D sur plusieurs TDM successives aboutissant à un panel de textures codé dans un logiciel ensuite automatisé [8, 34], soit directement via un logiciel 3D automatisé (e.g. CALIPER).

Le lien entre la fonction respiratoire et la densité pulmonaire a également été étudié, bien qu'il ne s'agisse pas de l'objectif principal de ce travail. Nos analyses montrent que la CVF et la CPT sont modérément et significativement corrélées à l'atteinte interstitielle fibrosante en TDM représentée par les voxels de haute densité. Ces constatations sont concordantes avec de précédents travaux montrant une corrélation inverse entre les lésions 
fibrosantes en TDM (\%HAA, \% d'atteinte réticulaire, scores de fibrose, MLD) et les paramètres fonctionnels de restriction, basés sur la $\operatorname{CVF}[6,7,35,36,37]$ et la CPT $[35,36]$ ainsi qu'une corrélation positive de la CVF avec le Kurtosis et le Skewness [6, 36], chez des patients présentant une PID. Dans ces mêmes études, les auteurs montraient également une corrélation entre densité pulmonaire et capacité de diffusion alvéolo-capillaire (DLCO), avec une relation inverse de ces 2 paramètres, que nous n'avons pas retrouvée dans ce travail. La densité pulmonaire était évaluée par différents paramètres assez comparables à ceux évalués par nos techniques d'analyse : le \%HAA, la MLD, le Kurtosis et le Skewness [6] et le pourcentage d'atteinte réticulaire [35]. Cette discordance avec nos résultats pourrait s'expliquer par des facteurs confondants à l'origine de variations de la DLCO. Il est en effet admis que la DLCO est liée à d'autres processus que l'atteinte interstitielle pulmonaire, dont, principalement, la présence d'une HTP [31] et le tabagisme [38]. La prévalence de l'HTP dans notre étude se trouve dans les tranches de prévalence décrites dans la littérature qui sont cependant très larges $(<15 \%$ à $>80 \%)$. De plus, cette prévalence n'est pas spécifiée dans les articles ayant étudiés la corrélation entre DLCO et paramètres tomodensitométriques. Nous ne rapportons pas non plus le statut tabagique des patients dans notre étude, ne permettant pas une comparaison ajustée.

Cette étude n'a pas démontré de lien entre la vascularisation pulmonaire de petite taille et l'HTP, contrairement à ce qui avait été démontré dans l'emphysème. Les trois études principales qui se sont intéressées à ce lien entre vascularisation $(\mathrm{CSA}<5)$ et $\mathrm{HTP}$, ont porté sur des populations de patients atteints d'emphysème ou d'HTP toutes causes confondues mal précisées. Elles s'étaient basées sur un seuil de -720UH pour présélectionner les zones contenant des vaisseaux, avant d'affiner les résultats sur la base de la circularité $[17,19,20]$. Dans ce travail, nous avons choisi un seuil un peu plus stringent de -600UH, comme utilisé précédemment dans l'étude de Froelich et coll. [39] portant sur l'analyse volumétrique de la vascularisation de petit calibre dans l'HTP, afin de s'affranchir d'un maximum de structures 
répondant à l'atteinte interstitielle (telles que les réticulations par ex.), tout en restant loin de la densité vasculaire définie, afin de ne pas sous-estimer la vascularisation. Ce changement de seuil peut expliquer la différence de nos résultats avec ceux de la littérature. Ce travail concernait, en effet, uniquement des patients atteints de PF-ILD sévère (statut pré-greffe pulmonaire), où la présence de lésions fibrosantes rendait la distinction de vaisseaux sur la base de la densité, plus difficile que dans d'autre pathologies telles que la BPCO ou même des PID modérées ou non évolutives, comme dans les 3 études rapportées précédemment. De surcroit, dans notre cohorte, le pourcentage important de territoires de destruction parenchymateuse à l'origine d'une raréfaction de la vascularisation a très probablement limité son analyse.

Cette étude a plusieurs limitations. Il s'agit d'une étude rétrospective observationnelle monocentrique de faible effectif. Notre population est en effet constituée de patients ayant tous par la suite eu une transplantation pulmonaire, ce qui nous permettait de confirmer le pattern évolutif fibrosant (histologie sur les poumons explantés). Cependant la perspective de transplantation générait un biais important de sélection, avec des patients, certes très sévères sur le plan de leur PID (atteinte interstitielle en progression), mais par ailleurs ayant peu ou pas de comorbidités, potentiellement plus jeunes que dans d'autres séries, et en particulier ayant peu ou pas d'HTP (objectivée par cathétérisme cardiaque droit systématique) car potentiellement récusés à la transplantation dans le cas contraire, ce qui limite l'extrapolation de nos résultats à l'ensemble des patients atteints de PF-ILD. Enfin, le fait d'avoir regroupé l'ensemble des patients atteints de PF-ILD rend notre cohorte hétérogène en termes de TDM, pouvant altérer en partie l'interprétation des paramètres de densité pulmonaire, en comparant des TDM dont le degré d'atteinte est différent, ou des FPI et des connectivites dont la physiopathologie est sûrement très différente.

Ainsi, nos résultats sont encore préliminaires et manquent pour l'heure de puissance statistique. Une confirmation sur un plus grand nombre de patients, inclus dans une étude 
prospective longitudinale, utilisant des algorithmes d'analyse TDM informatisés, permettant ainsi de s'affranchir de la variabilité inter-individuelle, devrait permettre d'accroître le niveau de preuve et conduire à une meilleure connaissance de l'étiopathogénie de la pathologie vasculaire (sévérité de l'hypertension pulmonaire) survenant dans le cours évolutif de la pathologie interstitielle pulmonaire.

\section{Conclusion}

L'exploitation de la tomodensitométrie, au-delà de son intérêt diagnostique, peut contribuer à une meilleure compréhension des différentes pathologies pulmonaires et constituer un réel modèle d'étude non invasif ayant pour avantage une approche pulmonaire totale in vivo.

Ce travail a permis de mettre en évidence, grâce à une technique d'analyse de densité en 3D de tomodensitométries thoraciques, un lien entre la densité pulmonaire et l'hypertension pulmonaire dans une cohorte de patients présentant une pneumopathie interstitielle diffuse d'évolution fibrosante. Il montre notamment que les patients atteints d'HTP sévère ont une atteinte fibrosante moins sévère que les patients avec HTP modérée ou sans HTP.

Ces résultats méritent d'être confirmés sur un plus grand nombre de patients dans une étude prospective longitudinale, permettant d'affiner l'analyse par l'individualisation des différentes structures propres à l'atteinte interstitielle fibrosante et à l'atteinte vasculaire pulmonaire dans cette pathologie, en utilisant des logiciels d'analyse tomodensitométrique de texture en 3D informatisés, ouvrant ainsi de larges perspectives diagnostiques, pronostiques et potentiellement thérapeutiques. 


\section{Références}

1. Cottin V, Wollin L, Fischer A, et al. Fibrosing interstitial lung diseases: knowns and unknowns. Eur Respir Rev 2019;28:180100.

2. Hayes D Jr, Black SM, Whitson BA, et al. Influence of Pulmonary Hypertension on Patients With Idiopathic Pulmonary Fibrosis Awaiting Lung Transplantation. Ann Thorac Surg 2016;101(1):246-52.

3. Corral-Blanco M, Prudencio-Ribera VC, Jarrín-Estupiñán ME, et al. Influence of Pulmonary Hypertension on Intrahospital Mortality in Lung Transplantation for Interstitial Lung Disease. Transplant Proc 2019;51(2):380-382.

4. Smith JS, Gorbett D, Mueller J, et al. Pulmonary hypertension and idiopathic pulmonary fibrosis: a dastardly duo. Am J Med Sci 2013;346(3):221-5.

5. Farkas L, Gauldie J, Voelkel NF, et al. Pulmonary hypertension and idiopathic pulmonary fibrosis: a tale of angiogenesis, apoptosis, and growth factors. Am J Respir Cell Mol Biol 2011;45(1):1-15.

6. Tanizawa K, Handa T, Nagai S, et al. Clinical impact of high-attenuation and cystic areas on computed tomography in fibrotic idiopathic interstitial pneumonias. BMC Pulm Med 2015;15:74.

7. Ash SY, Harmouche R, Vallejo DL, et al. Densitometric and local histogram based analysis of computed tomography images in patients with idiopathic pulmonary fibrosis. Respir Res 2017;18(1):45.

8. Kim HG, Tashkin DP, Clements PJ, et al. A computer-aided diagnosis system for quantitative scoring of extent of lung fibrosis in scleroderma patients. Clin Exp Rheumatol 2010;28(5 Suppl 62):S26-35. 
9. Best AC, Meng J, Lynch AM, et al. Idiopathic pulmonary fibrosis: physiologic tests, quantitative CT indexes, and CT visual scores as predictors of mortality. Radiology 2008;246(3):935-40.

10. Ryerson CJ, Urbania TH, Richeldi L, et al. Prevalence and prognosis of unclassifiable interstitial lung disease. Eur Respir J 2013;42(3):750-7.

11. Mogulkoc N, Brutsche MH, Bishop PW, et al. Pulmonary function in idiopathic pulmonary fibrosis and referral for lung transplantation. Am J Respir Crit Care Med 2001;164(1):103-8

12. Jacob J, Bartholmai BJ, Rajagopalan S, et al. Mortality prediction in idiopathic pulmonary fibrosis: evaluation of computer-based CT analysis with conventional severity measures. Eur Respir J 2017;49(1):1601011.

13. Jacob J, Bartholmai BJ, Rajagopalan S, et al. Unclassifiable-interstitial lung disease: Outcome prediction using CT and functional indices. Respir Med 2017;130:43-51.

14. De Giacomi F, Raghunath S, Karwoski R, et al. Short-term Automated Quantification of Radiologic Changes in the Characterization of Idiopathic Pulmonary Fibrosis Versus Nonspecific Interstitial Pneumonia and Prediction of Long-term Survival. J Thorac Imaging 2018;33(2):124-131.

15. Maldonado F, Moua T, Rajagopalan S, et al. Automated quantification of radiological patterns predicts survival in idiopathic pulmonary fibrosis. Eur Respir J 2014;43(1):20412.

16. Jacob J, Bartholmai BJ, Rajagopalan S, et al. Predicting Outcomes in Idiopathic Pulmonary Fibrosis Using Automated Computed Tomographic Analysis. Am J Respir Crit Care Med 2018;198(6):767-776. 
17. Matsuoka S, Washko GR, Dransfield MT, et al. Quantitative CT measurement of crosssectional area of small pulmonary vessel in COPD: correlations with emphysema and airflow limitation. Acad Radiol 2010;17(1):93-9.

18. Matsuura Y, Kawata N, Yanagawa N, et al. Quantitative assessment of cross-sectional area of small pulmonary vessels in patients with COPD using inspiratory and expiratory MDCT. Eur J Radiol 2013;82(10):1804-10.

19. Matsuoka S, Washko GR, Yamashiro T, et al. Pulmonary hypertension and computed tomography measurement of small pulmonary vessels in severe emphysema. Am J Respir Crit Care Med 2010;181(3):218-25.

20. Shimizu K, Tsujino I, Sato T, et al. Performance of computed tomography-derived pulmonary vasculature metrics in the diagnosis and haemodynamic assessment of pulmonary arterial hypertension. Eur J Radiol 2017;96:31-38.

21. Alhamad EH, Al-Boukai AA, Al-Kassimi FA, et al. Prediction of pulmonary hypertension in patients with or without interstitial lung disease: reliability of CT findings. Radiology 2011;260(3):875-83.

22. Zisman DA, Karlamangla AS, Ross DJ, et al. High-resolution chest CT findings do not predict the presence of pulmonary hypertension in advanced idiopathic pulmonary fibrosis. Chest 2007;132(3):773-9.

23. Hatano, Shuichi, Strasser, Toma \& World Health Organization. Primary pulmonary hypertension : report on a WHO meeting, Geneva, 15-17 October 1973 / edited by Shuichi Hatano and Toma Strasser. World Health Organization. (1975)

24. Mascalchi M, Camiciottoli G, Diciotti S. Lung densitometry: why, how and when. J Thorac Dis 2017;9(9):3319-3345.

25. Nathan SD, Shlobin OA, Ahmad S, et al. Pulmonary hypertension and pulmonary function testing in idiopathic pulmonary fibrosis. Chest 2007;131(3):657-663. 
26. Fartoukh M, Humbert M, Capron F, et al. Severe pulmonary hypertension in histiocytosis X. Am J Respir Crit Care Med. 2000 Jan;161(1):216-23.

27. Shorr AF, Helman DL, Davies DB, et al. Pulmonary hypertension in advanced sarcoidosis: epidemiology and clinical characteristics. Eur Respir J. 2005 May;25(5):783-8.

28. Hurdman J, Condliffe R, Elliot CA, et al. Pulmonary hypertension in COPD: results from the ASPIRE registry. Eur Respir J. 2013 Jun;41(6):1292-301.

29. Pugliese SC, Poth JM, Fini MA, et al. The role of inflammation in hypoxic pulmonary hypertension: from cellular mechanisms to clinical phenotypes. Am J Physiol Lung Cell Mol Physiol. 2015 Feb 1;308(3):L229-52.

30. Simonneau G, Montani D, Celermajer DS, et al. Haemodynamic definitions and updated clinical classification of pulmonary hypertension. Eur Respir J. 2019 Jan 24;53(1):1801913.

31. Lettieri CJ, Nathan SD, Barnett SD, et al. Prevalence and outcomes of pulmonary arterial hypertension in advanced idiopathic pulmonary fibrosis. Chest 2006;129:746752.

32. Shorr AF, Wainright JL, Cors CS, et al. Pulmonary hypertension in patients with pulmonary fibrosis awaiting lung transplant. Eur Respir J. 2007 Oct;30(4):715-21.

33. Nakagawa H, Nagatani Y, Takahashi M, et al. Quantitative CT analysis of honeycombing area in idiopathic pulmonary fibrosis: Correlations with pulmonary function tests. Eur J Radiol. 2016 Jan;85(1):125-130.

34. Ash SY, Harmouche R, Ross JC, Diaz AA, et al. The Objective Identification and Quantification of Interstitial Lung Abnormalities in Smokers. Acad Radiol. 2017 Aug;24(8):941-946. 
35. Bartholmai BJ, Raghunath S, Karwoski RA, et al. Quantitative computed tomography imaging of interstitial lung diseases. J Thorac Imaging. 2013 Sep;28(5):298-307.

36. Best AC, Lynch AM, Bozic CM, et al. Quantitative CT indexes in idiopathic pulmonary fibrosis: relationship with physiologic impairment. Radiology. 2003 Aug;228(2):40714.

37. Kim HJ, Brown MS, Chong D, et al. Comparison of the quantitative CT imaging biomarkers of idiopathic pulmonary fibrosis at baseline and early change with an interval of 7 months. Acad Radiol. 2015 Jan;22(1):70-80.

38. Tzani P, Aiello M, Colella M, et al. Lung Diffusion Capacity can Predict Maximal Exercise in Apparently Healthy Heavy Smokers. J Sports Sci Med. 2008 Jun 1;7(2):22934.

39. Froelich JJ, Koenig H, Knaak L, et al. Relationship between pulmonary artery volumes at computed tomography and pulmonary artery pressures in patients with- and without pulmonary hypertension. Eur J Radiol. 2008 Sep;67(3):466-71. 
Tableaux

Tableau 1 : Caractéristiques de la population de l'étude

\begin{tabular}{|c|c|c|c|c|c|}
\hline & & \multicolumn{3}{|c|}{ Groupes d'Hypertension Pulmonaire } & \\
\hline & Cohorte totale & $<25 \mathrm{mmHg}$ & $25-35 \mathrm{mmHg}$ & $>35 \mathrm{mmHg}$ & \\
\hline & $\mathrm{n}=57(100 \%)$ & $\mathrm{n}=20(35 \%)$ & $\mathrm{n}=33(58 \%)$ & $\mathrm{n}=4(7 \%)$ & $\mathrm{p}$ \\
\hline Age (années) médiane [IQR] & $61[55-64]$ & $58[54-61]$ & $61[57-64]$ & $64[61-65]$ & 0,12 \\
\hline Femmes, n (\%) & $16(28)$ & $10(63)$ & $6(37)$ & $\mathbf{0}(\mathbf{0})$ & $\mathbf{0 , 0 2}$ \\
\hline Poids (kg) & $74[66-82]$ & $74[59-80]$ & $75[68-83]$ & $68[65-71]$ & 0,21 \\
\hline Taille $(\mathrm{m})$ & $1,70[1,61-1,75]$ & $1,63[1,57-1,74]$ & $1,72[1,67-1,75]$ & $1,69[1,65-1,73]$ & 0,19 \\
\hline IMC $(\mathrm{kg} / \mathrm{m} 2)$ & $26,4[22,6-28,5]$ & $27,0[22,1-30,2]$ & $26,5[23,0-28,5]$ & $23,8[23,0-24,7]$ & 0,45 \\
\hline \multicolumn{6}{|l|}{ Diagnostic clinique } \\
\hline$F P I$ & $24(42)$ & $7(29)$ & $15(63)$ & $2(8)$ & 0,38 \\
\hline PINS idiopathique & $2(4)$ & $0(0)$ & $2(100)$ & $0(0)$ & \\
\hline Connectivites & $6(11)$ & $6(100)$ & $0(0)$ & $0(0)$ & \\
\hline Histiocytose & $1(2)$ & $0(0)$ & $1(100)$ & $0(0)$ & \\
\hline Pneumopathie d'hypersensibilité & $4(7)$ & $2(50)$ & $2(50)$ & $0(0)$ & \\
\hline$I P A F$ & $3(5)$ & $0(0)$ & $3(100)$ & $0(0)$ & \\
\hline Emphysème-Fibrose & $3(5)$ & $1(33)$ & $2(67)$ & $0(0)$ & \\
\hline Téloméropathie & $3(5)$ & $1(33)$ & $1(33)$ & $1(33)$ & \\
\hline Mutation SFTPA2 & $1(2)$ & $0(0)$ & $1(100)$ & $0(0)$ & \\
\hline Asbestose & $2(4)$ & $0(0)$ & $2(100)$ & $0(0)$ & \\
\hline Sarcoïdose & $1(2)$ & $0(0)$ & $1(100)$ & $0(0)$ & \\
\hline Sidérose & $1(2)$ & $0(0)$ & $1(100)$ & $0(0)$ & \\
\hline Inclassable & $6(11)$ & $3(50)$ & $2(33)$ & $1(17)$ & \\
\hline \multicolumn{6}{|l|}{ Cathétérisme cardiaque droit } \\
\hline PAPm $(\mathrm{mmHg})$ & $28[23-32]$ & $22[19-23]$ & $29[28-33]$ & $41[36-44]$ & - \\
\hline PAPs (mmHg) & $42[35-48]$ & 33 [29-37] & $45[40-49]$ & $64[59-74]$ & $<0,001$ \\
\hline$P A P d(m m H g)$ & $18[14-24]$ & $13[11-16]$ & $20[17-25]$ & $29[26-40]$ & $<0,001$ \\
\hline Pcap $(\mathrm{mmHg})$ & $12[8-14]$ & $12[8-14]$ & $12[8-14]$ & $12[8-14]$ & $\mathbf{0 , 0 3}$ \\
\hline Débit cardiaque $(\mathrm{L} / \mathrm{min})$ & $5,0[4,4-5,9]$ & $5,0[4,5-6,0]$ & $5,1[4,7-5,5]$ & $4,1[4,0-4,7]$ & 0,37 \\
\hline Index cardiaque $(\mathrm{L} / \mathrm{min} / \mathrm{m} 2)$ & $2,8[2,4-3,2]$ & $3,1[2,6-3,2]$ & $2,7[2,4-3,0]$ & $2,4[2,4-2,4]$ & 0,49 \\
\hline$R V P$ & $3,1[2,2-4,1]$ & $2,2[1,7-2,6]$ & $3,4[2,9-4,4]$ & $6,4[5,4-7,2]$ & $<0,001$ \\
\hline \multicolumn{6}{|l|}{ ETT } \\
\hline$P A P s(m m H g)$ & $35[30-52]$ & $35[25-35]$ & $37[30-53]$ & $55[45-87]$ & 0,12 \\
\hline$P O D(m m H g)$ & $5[3-5]$ & $3[3-5]$ & $5[5-5]$ & $5[4-12]$ & 0,52 \\
\hline TAPSE $(\mathrm{mm})$ & $21[18-24]$ & $22[20-24]$ & $21[19-24]$ & $18[15-22]$ & 0,47 \\
\hline Gradient VD-OD $(\mathrm{mmHg})$ & $30[25-50]$ & $21[21-22]$ & 30 [25-49] & $50[41-75]$ & 0,046 \\
\hline Cavités droites dilatées, $n(\%)$ & $10(18)$ & $2(10)$ & $5(15)$ & $3(75)$ & $\mathbf{0 , 0 1}$ \\
\hline Septum modifié, $n(\%)$ & $4(7)$ & $1(5)$ & $1(3)$ & $2(50)$ & 0,005 \\
\hline FEVD abaissée, n (\%) & $4(7)$ & $\mathbf{0}(\mathbf{0})$ & $2(6)$ & $2(50)$ & $\mathbf{0 , 0 0 3}$ \\
\hline $\mathrm{BNP}$ & 19 [12-35] & $15[10-32]$ & 20 [12-36] & $14[12-175]$ & 0,53 \\
\hline \multicolumn{6}{|l|}{ EFR } \\
\hline CVF (\%théo) & 47 [39-54] & $44[35-51]$ & $48[40-56]$ & $64[54-72]$ & 0,05 \\
\hline CPT (\%théo) & $51[46-55]$ & $48[44-52]$ & $52[46-55]$ & $60[53-70]$ & 0,10 \\
\hline VEMS/CVL (ratio) & $0,85[0,81-0,92]$ & $0,90[0,82-0,95]$ & $0,85[0,80-0,90]$ & $0,81[0,76-0,84]$ & 0,11 \\
\hline DLCO (\%théo) & $18[15-24]$ & $21[16-24]$ & $16[12-24]$ & $17[17-20]$ & 0,53 \\
\hline KCO (\%théo) & $41[33-49]$ & $46[36-51]$ & $41[28-48]$ & $36[35-38]$ & 0,44 \\
\hline \multicolumn{6}{|l|}{ Gaz du sang } \\
\hline $\mathrm{pH}$ & $7,43[7,41-7,45]$ & $7,43[7,41-7,44]$ & $7,43[7,42-7,45]$ & $7,42[7,42-7,44]$ & 0,85 \\
\hline $\mathrm{PaCO} 2(\mathrm{mmHg})$ & $39[36-44]$ & $39[38-43]$ & $40[36-45]$ & $33[31-37]$ & 0,27 \\
\hline $\mathrm{HCO} 3-(\mathrm{mmol} / \mathrm{L})$ & $25[24-28]$ & $25[23-28]$ & $25[24-31]$ & $22[22-22]$ & 0,97 \\
\hline $\mathrm{O} 2$ débit $(\mathrm{L} / \mathrm{min})$ & $3[2-4]$ & $3[2-4]$ & $3[3-4]$ & $2[2-2]$ & 0,60 \\
\hline $\mathrm{PaO} 2(\mathrm{mmHg})$ & 63 [55-79] & $64[57-75]$ & $62[54-80]$ & $61[58-73]$ & 0,97 \\
\hline \multicolumn{6}{|l|}{ TM6 } \\
\hline Distance $(\mathrm{m})$ & $380[275-425]$ & 371 [280-406] & $390[237-441]$ & $360[323-402]$ & 0,76 \\
\hline $\mathrm{O} 2$ débit $(\mathrm{L} / \mathrm{min})$ & $7[6-9]$ & $6[5-7]$ & $7[6-11]$ & $7[5-10]$ & 0,27 \\
\hline $\mathrm{SpO} 2$ début & 97 [96-98] & 97 [95-97] & 97 [96-97] & 97 [97-98] & 0,54 \\
\hline $\mathrm{SpO} 2 \mathrm{fin}$ & $83[79-88]$ & $82[80-86]$ & $83[79-88]$ & $82[80-84]$ & 0,64 \\
\hline \multicolumn{6}{|l|}{ Traitements } \\
\hline Antihypertenseur pulmonaire, n (\%) & $3(5)$ & $\mathbf{0}(\mathbf{0})$ & $\mathbf{0}(\mathbf{0})$ & $3(75)$ & $<0,001$ \\
\hline Anti-aggrégant plaquettaire, $\mathrm{n}(\%)$ & $20(35)$ & $7(35)$ & $10(30)$ & $3(75)$ & 0,47 \\
\hline
\end{tabular}




\begin{tabular}{|r|c|c|c|c|c|} 
Anticoagulant, $\mathrm{n}(\%)$ & $3(5)$ & $0(0)$ & $2(10)$ & $1(25)$ & 0,13 \\
\hline Diurétique, n (\%) & $\mathbf{1 5}(\mathbf{2 6})$ & $\mathbf{1}(\mathbf{5})$ & $\mathbf{1 0}(\mathbf{3 0})$ & $\mathbf{4}(\mathbf{1 0 0})$ & $\mathbf{0 , 0 0 1}$ \\
\hline Inhibiteur calcique & $5(9)$ & $0(0)$ & $5(15)$ & $0(0)$ & 0,13 \\
\hline Anti-fibrotique & $12(21)$ & $2(10)$ & $9(27)$ & $1(25)$ & 0,35 \\
\hline Immunosuppresseur & $40(70)$ & $17(85)$ & $21(63)$ & $2(50)$ & 0,05 \\
\hline O2 & $57(100)$ & $20(100)$ & $33(100)$ & $4(100)$ & 1,00 \\
\hline O2 débit (L/min) & $3[2-4]$ & $3[2-4]$ & $4[2-4]$ & $3[3-5]$ & 0,81 \\
\hline Temps entre TDM et KTd (jours) & $7[2-50]$ & $18[3-47]$ & $6[2-49]$ & $3[2-33]$ & 0,74 \\
\hline
\end{tabular}

Les paramètres différant de manière statistiquement significative $(\mathrm{p}<0.05)$ entre les 3 groupes d'hypertension pulmonaire (HTP) sont représentés en gras. Les variables quantitatives sont représentées en médiane et interquartiles (entre crochets).

IMC = Indice de masse corporelle $;$ FPI = Fibrose pulmonaire idiopathique $;$ PINS = Pneumopathie interstitielle non spécifique ; IPAF = Pneumopathie interstitielle avec terrain d'auto-immunité ; PAPm = Pression artérielle pulmonaire moyenne $;$ PAPs $=$ Pression artérielle pulmonaire systolique $;$ PAPd $=$ Pression artérielle pulmonaire diastolique $;$ Pcap = pression capillaire $;$ RVP = Résistances vasculaires pulmonaires $;$ ETT = Echocardiographie transthoracique $;$ POD $=$ Pression de l'oreillette droite $;$ TAPSE $=$ Excursion systolique du plan de l'anneau tricuspide $; \mathrm{FEVD}=$ Fraction d'éjection du ventricule droit $; \mathrm{BNP}=$ peptide natriurétique cérébral ; Scintigraphie $\mathrm{V} / \mathrm{P}=$ scintigraphie de ventilation/perfusion $; \mathrm{EFR}=$ Epreuve fonctionnelle respiratoire $; \mathrm{CVL}=\mathrm{Capacité} \mathrm{vitale}$ lente $; \mathrm{CPT}=$ Capacité pulmonaire totale $; \mathrm{VEMS}=$ Volume expiratoire maximal seconde $;$ DLCO = capacité de diffusion du monoxyde de carbone; $\mathrm{KCO}=$ coefficient de diffusion du monoxyde de carbone ; TM6 = test de marche de 6 minutes ; TDM = tomodensitométrie $; \mathrm{KTd}=$ cathétérisme cardiaque droit 
Tableau 2 : Corrélation entre densité pulmonaire, vascularisation et hypertension pulmonaire

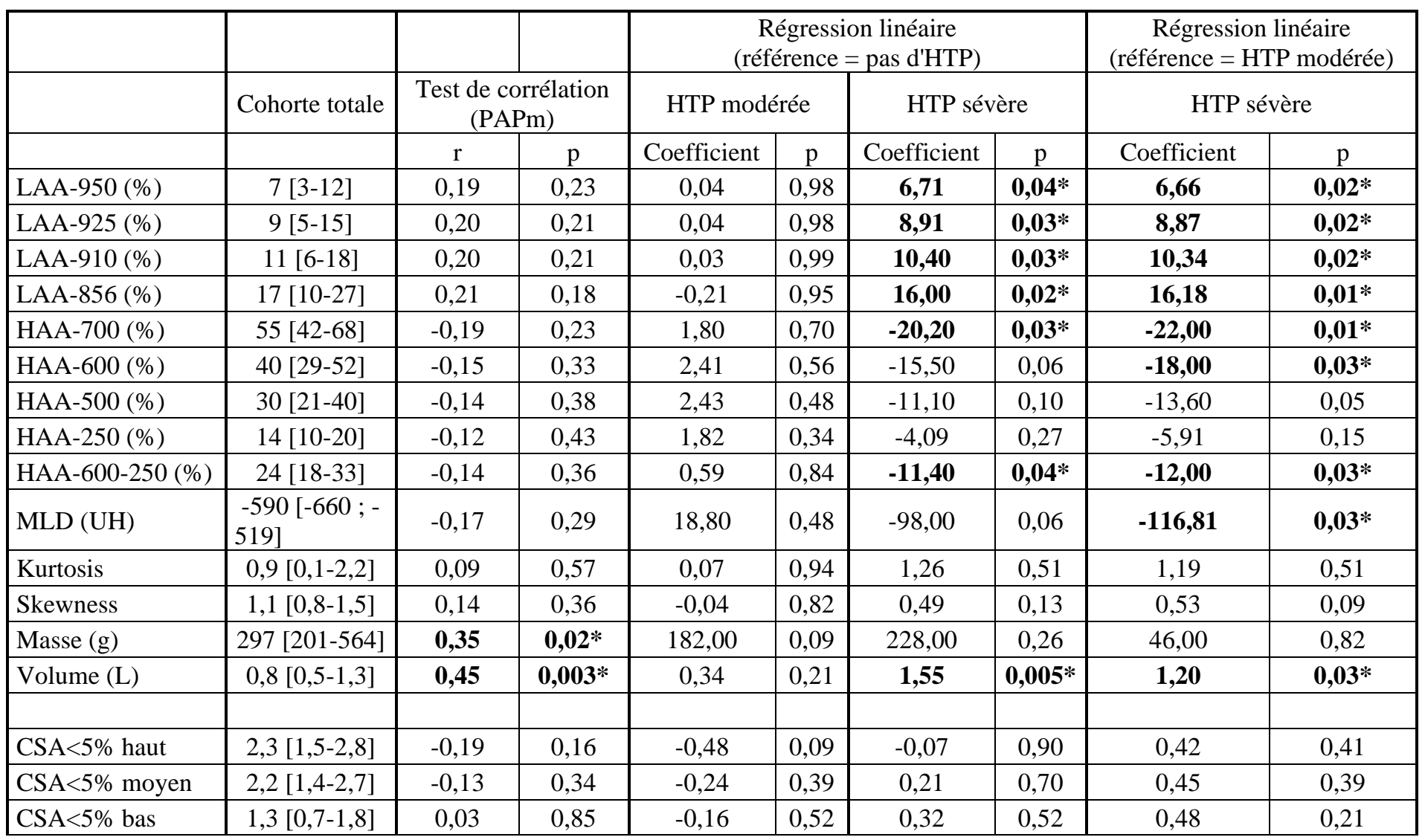

En gras sont représentés les coefficients statistiquement significatifs. *p<0.05

HTP $=$ Hypertension pulmonaire $;$ PAPm = Pression artérielle pulmonaire moyenne ; LAA = Low attenuation area $=\%$ voxels de faible densité $;$ HAA $=$ High attenuation area $=\%$ voxels de haute densité $;$ MLD $=$ mean lund density $=$ densité pulmonaire moyenne $; \mathrm{UH}=$ Unité Hounsfield ; CSA $<5=$ cross sectional area $=\%$ surface des vaisseaux $<5 \mathrm{~mm}^{2} /$ surface pulmonaire totale 
Tableau 3 : Corrélation entre densité pulmonaire, vascularisation et fonction respiratoire

\begin{tabular}{|c|c|c|c|c|c|c|}
\hline & \multicolumn{2}{|c|}{ CVF (\%théo) } & \multicolumn{2}{|c|}{ CPT (\%théo) } & \multicolumn{2}{|c|}{ DLCO (\%thé) } \\
\hline & $\mathrm{r}$ & $\mathrm{p}$ & $\mathrm{r}$ & $\mathrm{p}$ & $\mathrm{r}$ & $\mathrm{p}$ \\
\hline LAA-950 (\%) & 0,42 & 0,006* & 0,48 & 0,002* & $-0,10$ & 0,64 \\
\hline LAA-925 (\%) & 0,44 & $\mathbf{0 , 0 0 4 *}$ & 0,48 & $\mathbf{0 , 0 0 1 *}$ & $-0,09$ & 0,67 \\
\hline LAA-910 (\%) & 0,45 & $0,003 *$ & 0,48 & $0,002 *$ & $-0,08$ & 0,68 \\
\hline LAA-856 (\%) & 0,47 & 0,002* & 0,46 & 0,003* & $-0,18$ & 0,71 \\
\hline HAA-700 (\%) & $-0,41$ & 0,008* & $-0,40$ & 0,009* & 0,09 & 0,64 \\
\hline HAA-600 (\%) & $-0,37$ & $\mathbf{0 , 0 2 *}$ & $-0,39$ & 0,01* & 0,12 & 0,57 \\
\hline HAA-500 (\%) & $-0,37$ & $0,02 *$ & $-0,39$ & $0,01 *$ & 0,14 & 0,50 \\
\hline HAA-250 (\%) & $-0,36$ & $0,02 *$ & $-0,38$ & $0,01 *$ & 0,13 & 0,52 \\
\hline HAA-600-250 (\%) & $-0,31$ & 0,049* & $-0,32$ & 0,04* & 0,09 & 0,67 \\
\hline MLD (HU) & $-0,43$ & $0,005^{*}$ & $-0,43$ & 0,004* & 0,06 & 0,76 \\
\hline Kurtosis & $\mathbf{0 , 4 1}$ & 0,008* & 0,46 & 0,003* & $-0,09$ & 0,67 \\
\hline Skewness & 0,46 & $\mathbf{0 , 0 0 3 *}$ & $\mathbf{0 , 5 1}$ & $<0,001 *$ & $-0,17$ & 0,41 \\
\hline Masse (g) & 0,16 & 0,33 & 0,07 & 0,68 & $-0,13$ & 0,52 \\
\hline Volume (L) & 0,51 & $<0,001 *$ & 0,42 & 0,006* & $-0,19$ & 0,36 \\
\hline CSA5\% haut & $-0,06$ & 0,66 & 0,12 & 0,37 & $-0,11$ & 0,51 \\
\hline CSA5\% moyen & $-0,02$ & 0,87 & 0,14 & 0,29 & $-0,14$ & 0,42 \\
\hline CSA5\% bas & 0,19 & 0,16 & 0,23 & 0,08 & $-0,06$ & 0,71 \\
\hline
\end{tabular}

En gras sont représentés les coefficients statistiquement significatifs. *p $<0.05$

$\mathrm{CPT}=$ Capacité pulmonaire totale $; \mathrm{KCO}=$ coefficient de diffusion du monoxyde de carbone $; \mathrm{UH}=$ Unité Hounsfield $; \mathrm{LAA}=$ Low attenuation area $=\%$ voxels de faible densité $; \mathrm{HAA}=$ High attenuation area $=\%$ voxels de haute densité $; \mathrm{MLD}=$ mean lung density $=$ densité pulmonaire moyenne $; \mathrm{UH}=$ Unité Hounsfield $; \mathrm{CSA}<5=$ cross sectional area $=\%$ surface des vaisseaux $<5 \mathrm{~mm}^{2} /$ surface pulmonaire totale 


\section{Légendes des figures}

Figure 1: Représentation d'une reconstruction 3D de l'arbre trachéo-bronchique et des poumons à partir d'une tomodensitométrie thoracique, avec segmentation des lobes. Pour l'analyse, les grosses voies aériennes étaient exclues à l'aide d'un module de segmentation automatisé.

Figure 2 : Échelle de gris (grayscale) correspondant aux différentes unités Hounsfield (UH) de -1000 à +1000 , permettant d'estimer la densité (en UH) à partir des pixels d'une image ou des voxels d'un volume.

Figure 3: Représentation d'un histogramme de poumon normal à partir des données tomodensitométriques ayant fait l'objet d'une analyse quantitative dans le logiciel 3D slicer. L'histogramme en noir représente la fréquence de voxels (en \% des voxels totaux) pour chaque UH. Les voxels correspondant aux UH inférieures à -856 donc aux UH de faible densité (LAA$856 \%$ ) sont représentés par l'aire sous la courbe en bleu. Dans cet exemple, le LAA -856\% est de $56 \%$, soit $56 \%$ des voxels totaux ont une densité <-856 UH. Les voxels correspondant aux UH supérieures à -600 donc aux UH de haute densité (HAA-600\%) sont représentés par l'aire sous la courbe en rouge. Dans cet exemple, le HAA-600\% est de 6\%, soit 6\% des voxels totaux ont une densité > -600UH.

Figure 4: Représentation d'un histogramme de densité à partir de l'analyse de tomodensitométries pulmonaires d'un individu sain avec poumon normal (en noir) et d'un individu atteint de PF-ILD (en rouge). La zone grise représente la différence d'aire sous la courbe entre les deux histogrammes. Le coefficient appelé Kurtosis est le coefficient d'aplatissement de la courbe de l'histogramme. On remarque que plus le Kurtosis est bas, plus le sommet de la courbe est bas et l'aspect est aplati. Le coefficient appelé Skewness représente le coefficient d'asymétrie de répartition par rapport à une distribution normale. Le Skewness nul (représenté par la ligne grise en pointillés) représente la médiane d'une courbe théorique de distribution normale. On remarque que plus le Skewness est proche de 0, plus la répartition des valeurs tend vers une distribution normale symétrique. Dans cet exemple, le Skewness reste positif car la distribution est décalée à gauche de la médiane normale. Ce schéma met en évidence la différence entre un poumon sain et un poumon atteint de PF-ILD : lorsqu'une atteinte fibrosante est présente, les coefficients de Kurtosis et Skewness diminuent, donnant un aspect plus aplati et de distribution moins asymétrique. Cette modification de la répartition est en lien avec la modification de la proportion d'UH de faible densité qui diminue (dans cet exemple, le LAA- $856 \%$ est de $56 \%$ pour le poumon sain et $24 \%$ pour le poumon atteint de PFILD) et celle d'UH de haute densité qui augmente (dans cet exemple, le HAA-600\% est de 6\% pour le poumon sain et $30 \%$ pour le poumon atteint de PF-ILD) de façon proportionnelle à l'atteinte fibrosante.

Figure 5 : Représentation du processus de quantification vasculaire pulmonaire totale en 2D (logiciel Image J). A. Sélection de coupes axiales sur 3 niveaux (haut, moyen, bas) B. Pour chaque coupe, sélection automatique du parenchyme pulmonaire (contours en jaune) $\mathbf{C}$. 
Extraction de la zone d'intérêt (parenchyme pulmonaire) et calcul de la surface pulmonaire D. Application d'un filtre ne conservant que les UH > - 600 UH E. Transformation en image binaire F. Sélection des vaisseaux pulmonaires sur la base de leur circularité (0,9-1). L'image représente les vaisseaux de diamètre $<5 \mathrm{~mm} 2$. La surface totale vasculaire est ensuite rapportée à la surface parenchymateuse totale (\%CSA).

Figure 6: Histogramme de répartition des densités (UH) à partir de tomodensitométries pulmonaires de patients atteints de pneumopathie interstitielle diffuse d'évolution fibrosante (PF-ILD). Chaque courbe représente la moyenne du groupe de fréquence en \% de voxels totaux de chaque UH, au sein des 3 groupes d'individus 1) sans hypertension pulmonaire (HTP) (en vert), 2) HTP modérée (en gris) et 3) HTP sévère (en rouge). A noter un moindre aplatissement (kurtosis plus élevé) et une plus grande asymétrie de répartition vers la gauche (skewness plus élevé) chez les patients présentant une HTP sévère par rapport aux deux autres groupes.

Figure 7 : Représentation sous forme de nuage de points de la corrélation entre la capacité pulmonaire totale (CPT) en litres mesurée lors d'une épreuve fonctionnelle respiratoire et le volume pulmonaire en litres estimé par le logiciel d'analyse 3D slicer à partir d'une tomodensitométrie thoracique. On note l'existence d'une corrélation importante $(r=0,86)$ et significative $(\mathrm{p}<0.001)$ entre ces deux paramètres. 


\section{Figures}

Figure 1 :

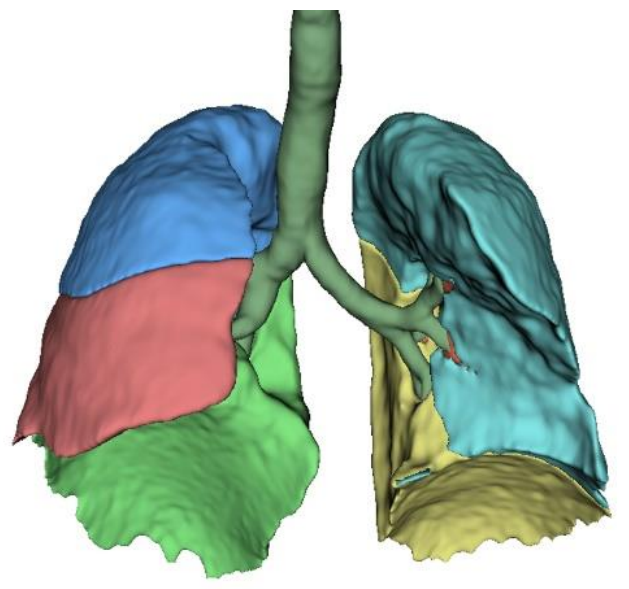

FPS: $106004021303 \mathrm{~s}$

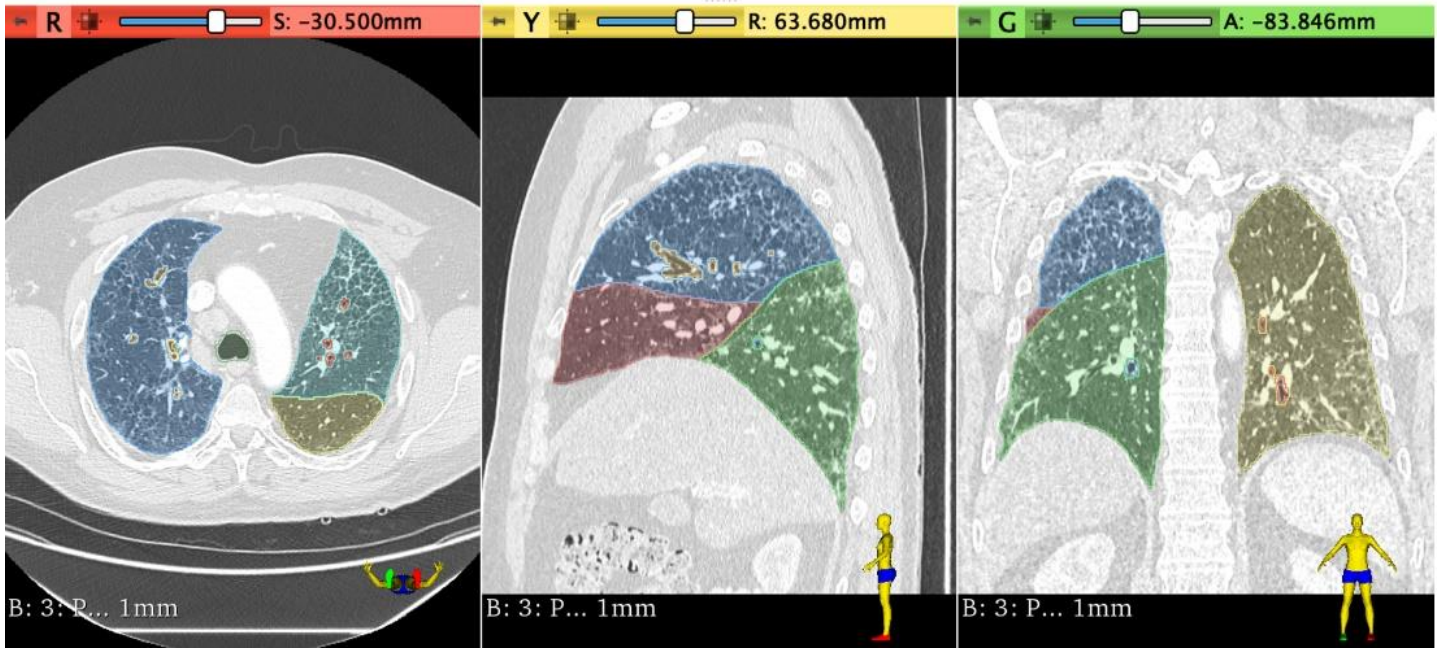


Figure 2 :

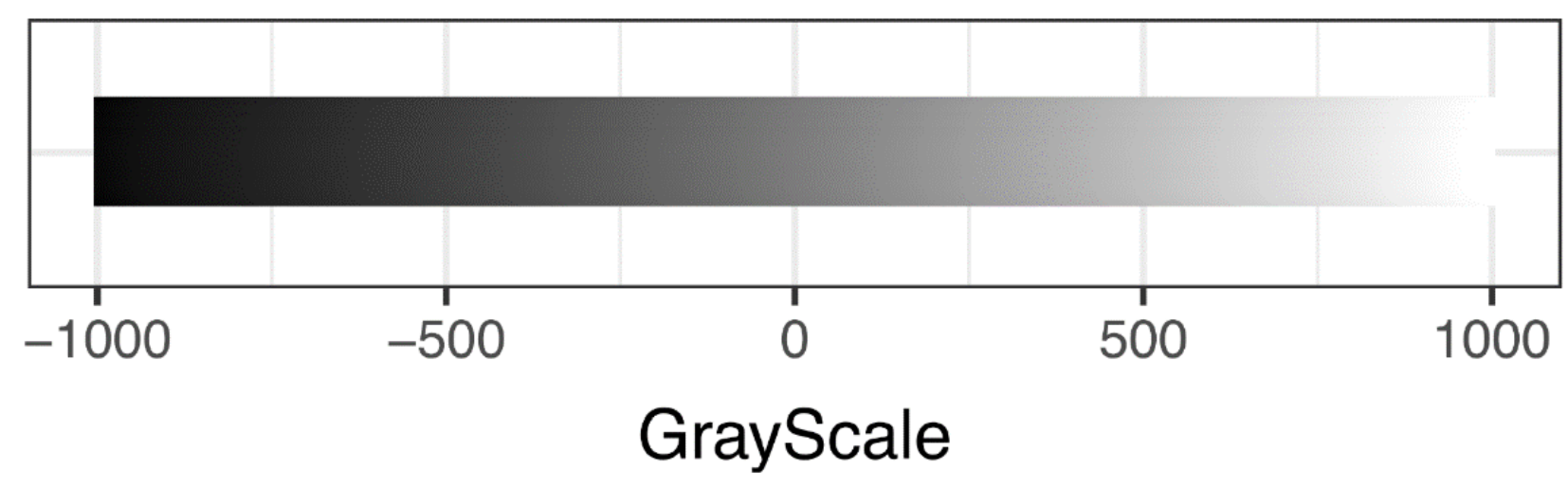


Figure 3 :

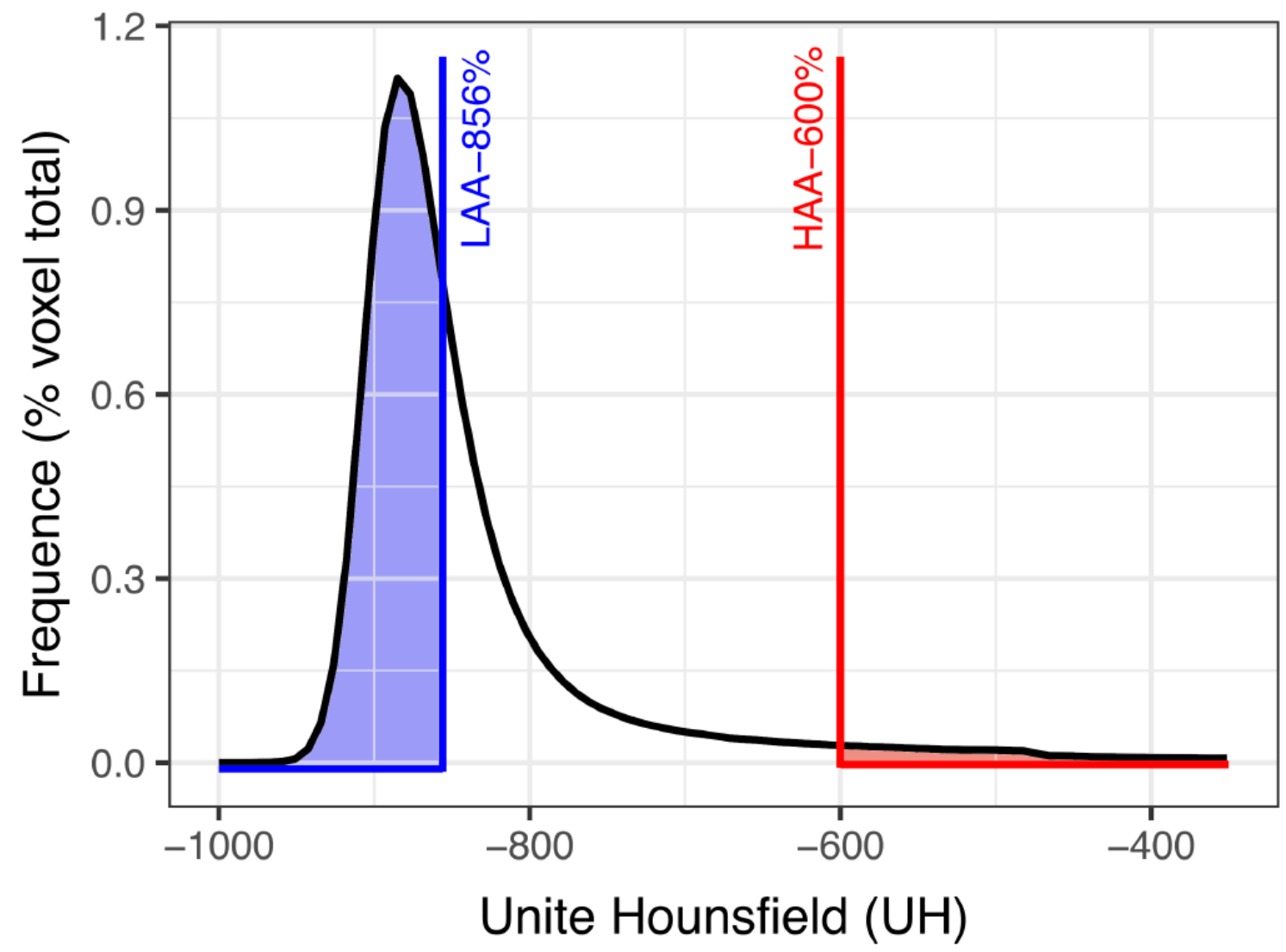


Figure 4 :

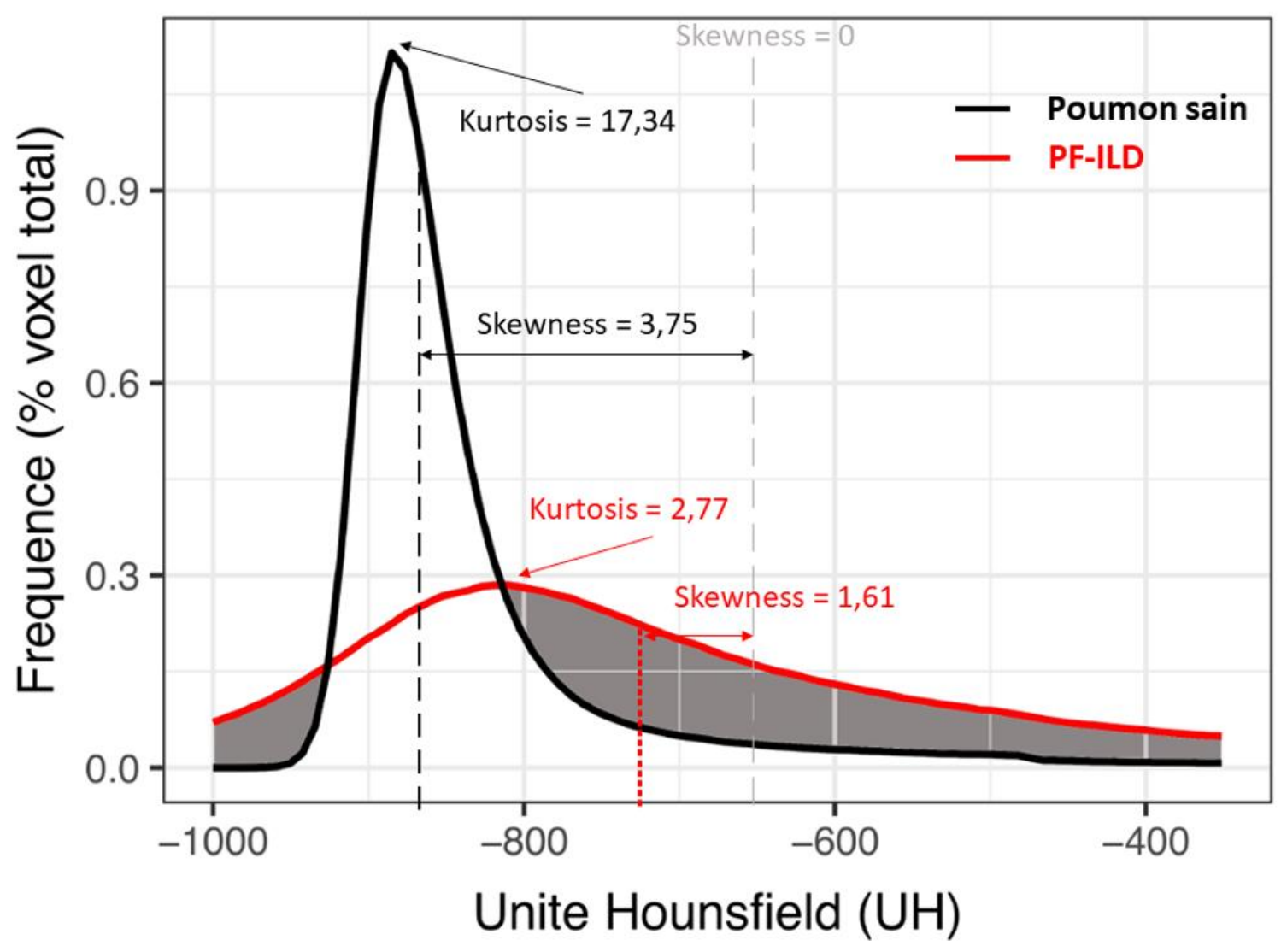


Figure 5 :
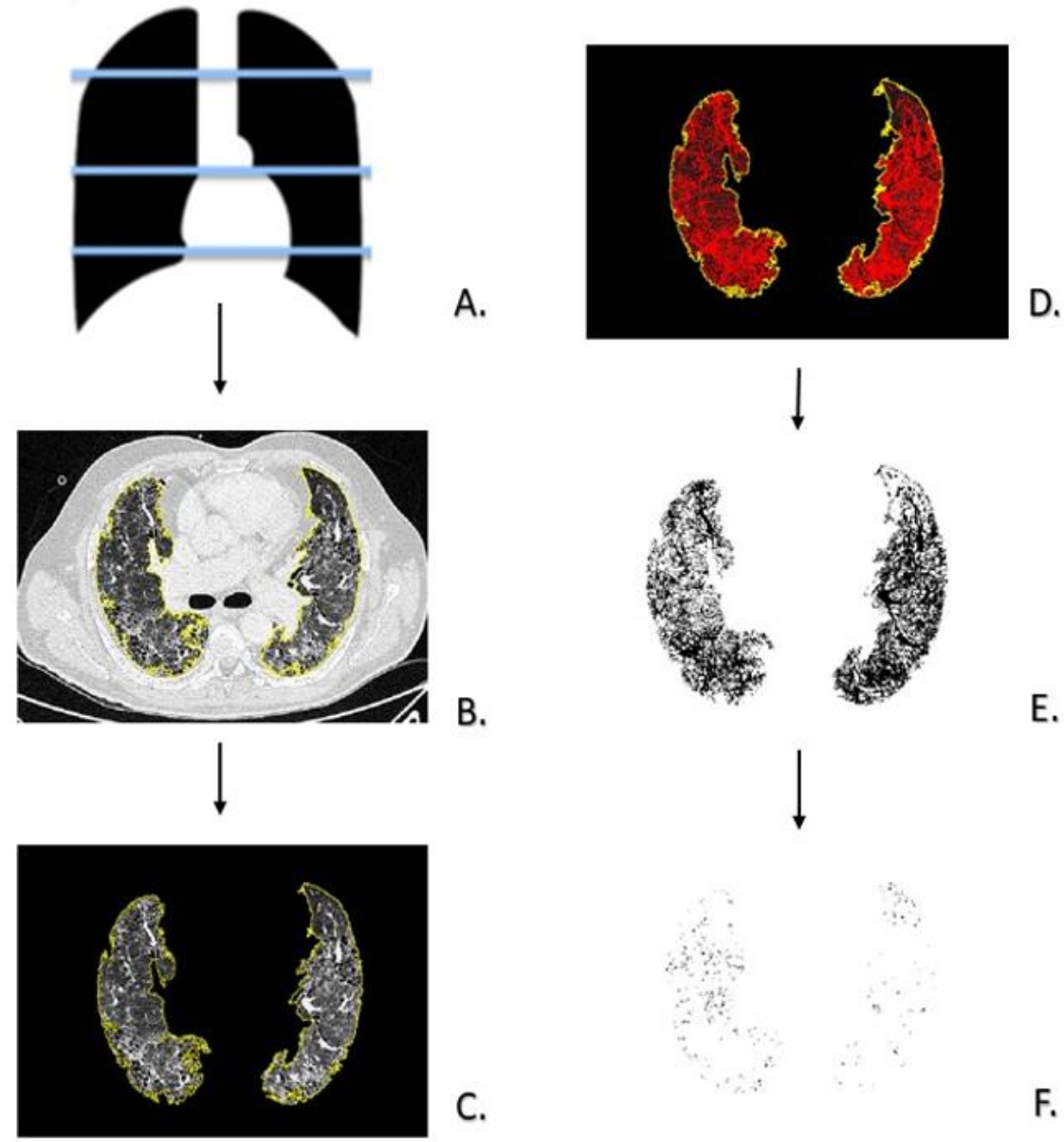

E.

C.

F. 
Figure 6 :

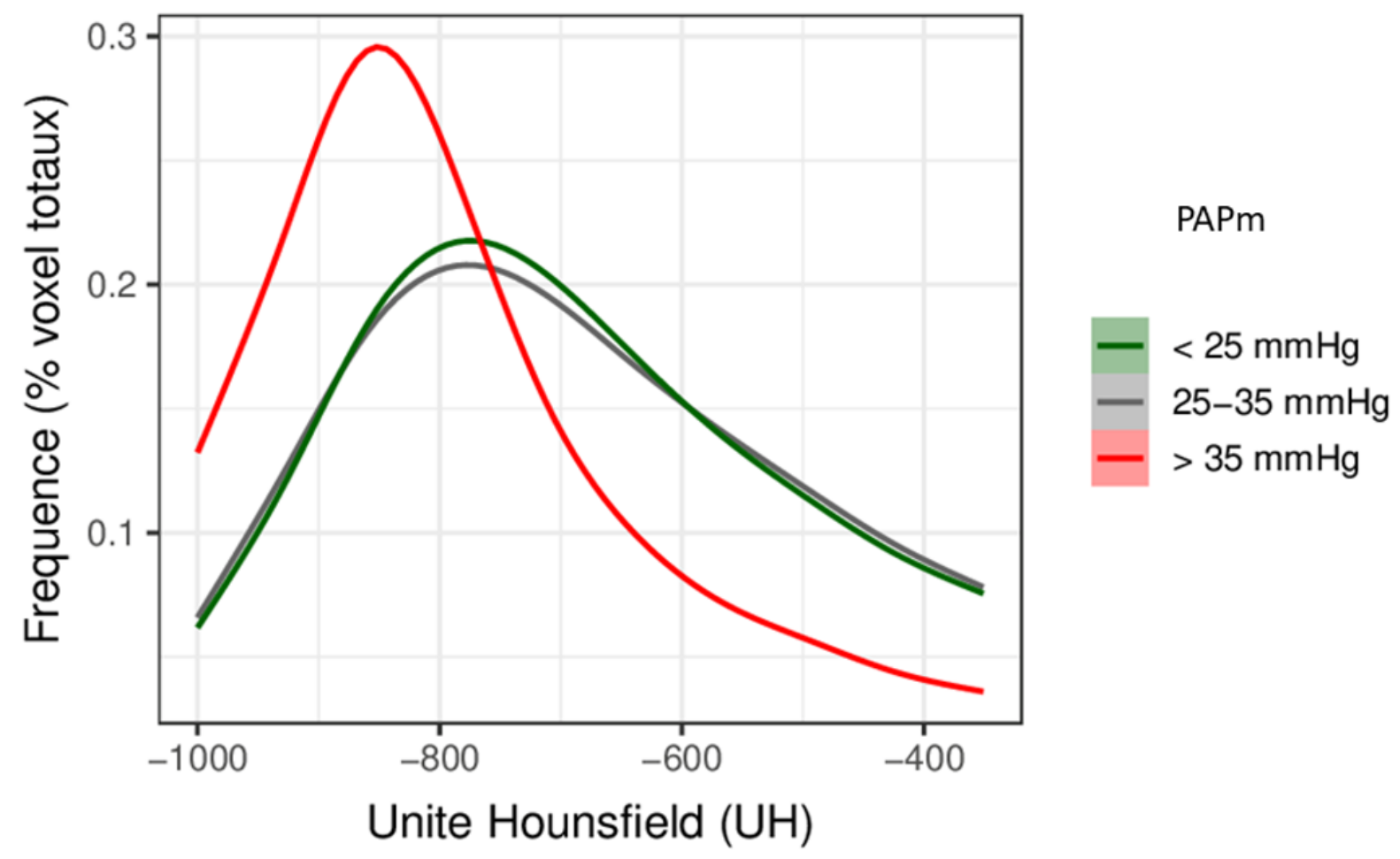


Figure 7 :

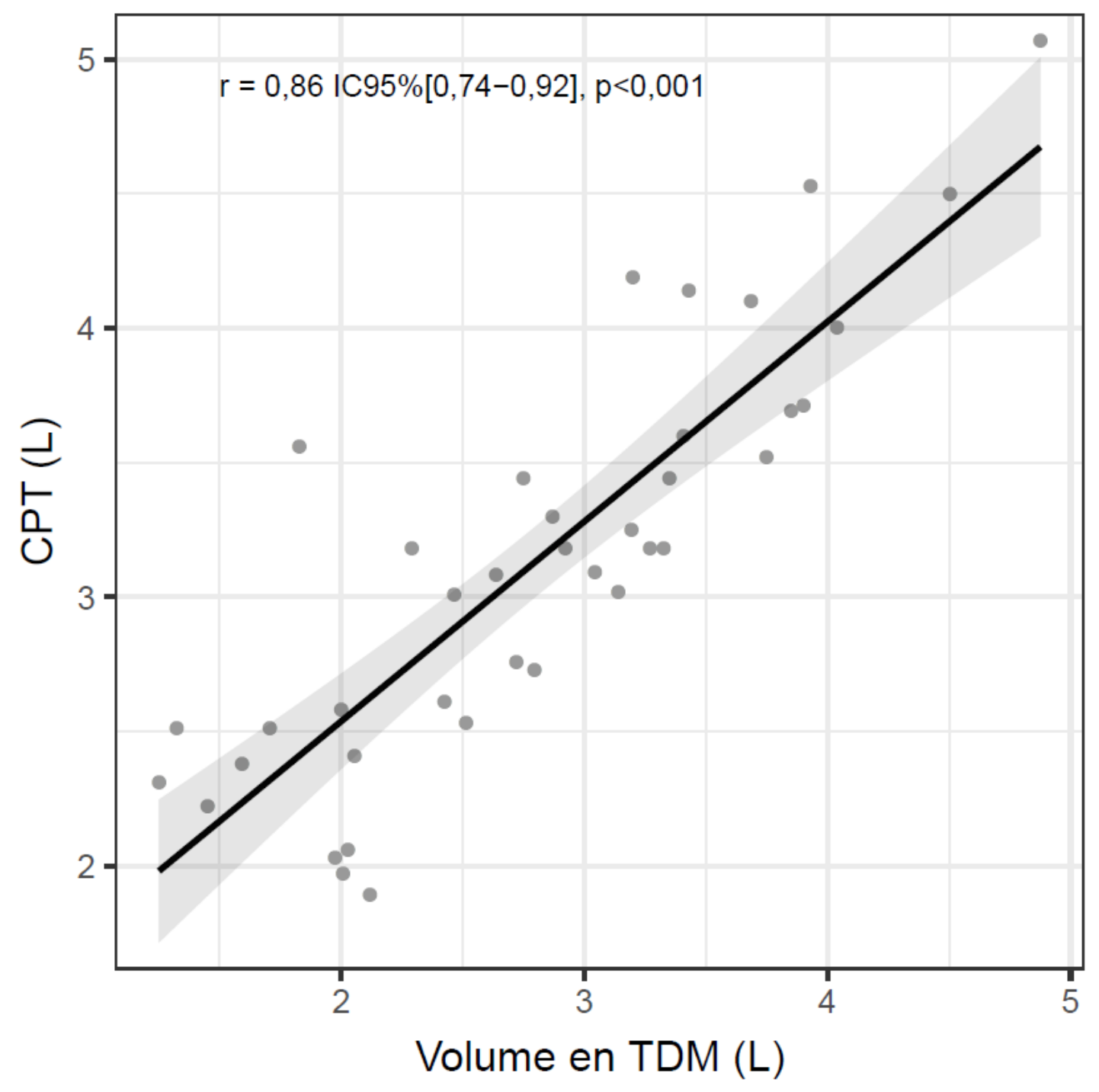




\section{SERMENT D'HIPPOCRATE}

Au moment d'être admis(e) à exercer la médecine, je promets et je jure d'être fidèle aux lois de l'honneur et de la probité.

Mon premier souci sera de rétablir, de préserver ou de promouvoir la santé dans tous ses éléments, physiques et mentaux, individuels et sociaux.

Je respecterai toutes les personnes, leur autonomie et leur volonté, sans aucune discrimination selon leur état ou leurs convictions. J'interviendrai pour les protéger si elles sont affaiblies, vulnérables ou menacées dans leur intégrité ou leur dignité. Même sous la contrainte, je ne ferai pas usage de mes connaissances contre les lois de l'humanité.

J'informerai les patients des décisions envisagées, de leurs raisons et de leurs conséquences.

Je ne tromperai jamais leur confiance et n'exploiterai pas le pouvoir hérité des circonstances pour forcer les consciences.

Je donnerai mes soins à l'indigent et à quiconque me les demandera. Je ne me laisserai pas influencer par la soif du gain ou la recherche de la gloire.

Admis(e) dans l'intimité des personnes, je tairai les secrets qui me seront confiés. Reçu(e) à l'intérieur des maisons, je respecterai les secrets des foyers et ma conduite ne servira pas à corrompre les moeurs.

Je ferai tout pour soulager les souffrances. Je ne prolongerai pas abusivement les agonies. Je ne provoquerai jamais la mort délibérément.

Je préserverai l'indépendance nécessaire à l'accomplissement de ma mission. Je n'entreprendrai rien qui dépasse mes compétences. Je les entretiendrai et les perfectionnerai pour assurer au mieux les services qui me seront demandés.

J'apporterai mon aide à mes confrères ainsi qu'à leurs familles dans l'adversité.

Que les hommes et mes confrères m'accordent leur estime si je suis fidèle à mes promesses ; que je sois déshonoré(e) et méprisé(e) si j'y manque.

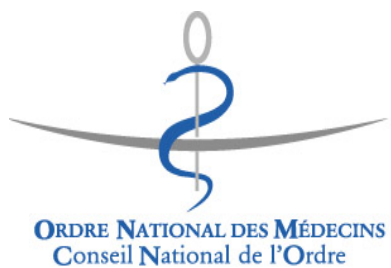





\section{Résumé}

Introduction : L'hypertension pulmonaire (HTP) associée aux pathologies respiratoires est encore mal comprise. L'objectif principal était d'évaluer la relation entre tomodensitométrie (TDM) pulmonaire et HTP chez des patients atteints de pneumopathie interstitielle diffuse d'évolution fibrosante (PF-ILD).

Méthodes : L'étude a porté sur des PF-ILD, ayant tous bénéficié d'une TDM et d'un cathétérisme cardiaque droit avant transplantation pulmonaire. Les TDM ont fait l'objet d'une analyse quantitative de la densité et de la vascularisation pulmonaire. La relation entre TDM et HTP a été étudiée.

Résultats : 57 patients ont été inclus. La PAPm était corrélée avec la masse pulmonaire $(\mathrm{r}=0,35 ; \mathrm{p}=0,02)$ et le volume pulmonaire $(\mathrm{r}=0,45 ; \mathrm{p}=0,003)$. Les patients avec HTP sévère avaient plus de voxels d'unité Hounsfield (UH) inférieure à $-856(+16 \% ; p=0,02)$, moins de voxels d'UH supérieure à $-700(-20 \%$; $\mathrm{p}=0,03)$, et un volume pulmonaire plus élevé $(+1,55 \mathrm{~L}$, p=0,005) comparés à ceux sans HTP. Aucune corrélation n’a été retrouvée entre vascularisation et HTP.

Conclusions : L'analyse TDM quantitative permet d'établir un lien entre densité parenchymateuse et la présence d'une HTP dans la PF-ILD. Les patients atteints avec HTP sévère ont des atteintes interstitielles moins marquées que les patients avec HTP modérée ou pas d'HTP.

Mots-clés : Pneumopathie interstitielle idiopathique ; Fibrose pulmonaire ; Hypertension pulmonaire ; Tomodensitométrie ; Raréfaction de la vascularisation. 